\title{
Asymmetric Synthesis of the AB Ring System of Lactonamycin
}

\author{
T. Ross Kelly,* Xiaolu Cai, Bin Tu, Eric L. Elliott, Gilles Grossmann, Pierre Laurent. \\ E. F. Merkert Chemistry Center, Boston College, Chestnut Hill, Massachusetts 02467 \\ Supporting Information
}

ross.kelly@bc.edu

\section{Table of Contents}

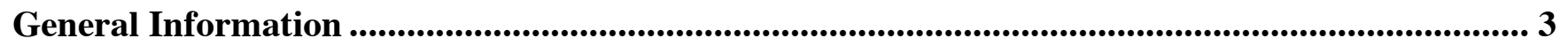

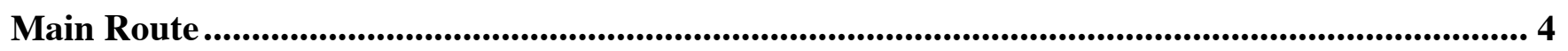

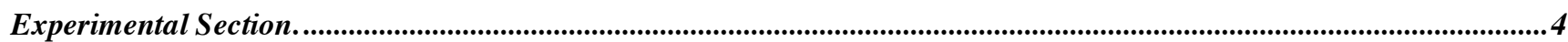

(2S)-(-)-2-Benzyloxymethyl-2,3,3-tris(methoxycarbonyl)-1,4-dioxaspiro[4.4] nonane (32)............................................

(3R-cis)-(+)-Dihydro-3,4-dihydroxy-3,4-bis(methoxycarbonyl)furan-2(3H)-one (38)...................................................4

(3S-trans)-(-)-Dihydro-3,4-dihydroxy-3,4-bis(methoxycarbonyl)furan-2(3H)-one (39)................................................5

(2S)-2-Benzyloxymethyl-2,3-dihydroxy-3-methoxycarbonylsuccinic acid dimethyl ester (40)......................................5

(2R,3aR-cis)-(+)-Dihydro-3a,6a-bis(methoxycarbonyl)-2-phenylfuro[3,4-d][1,3]dioxol-4(3aH)-one (46)......................5

(2S,3aR-cis)-(+)-Dihydro-3a,6a-bis(methoxycarbonyl)-2-phenylfuro[3,4-d][1,3]dioxol-4(3aH)-one (47)......................6

$(2 R, 3 a R, 6 a S)-(+)-4$-tert-Butoxycarbonylmethyl-3a,6a-bis(methoxycarbonyl)-2-

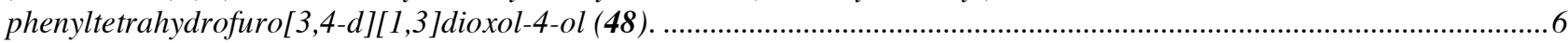

(2S,3aR,6aS)-(+)-4-tert-Butoxycarbonylmethyl-3a,6a-bis(methoxycarbonyl)-2-

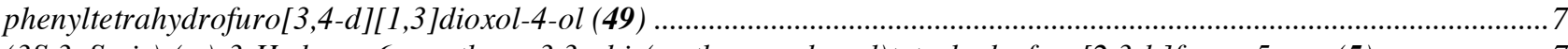

(3S,3aS-cis)-(+)-3-Hydroxy-6a-methoxy-3,3a-bis(methoxycarbonyl)tetrahydrofuro[2,3-b]furan-5-one (5).................... 7

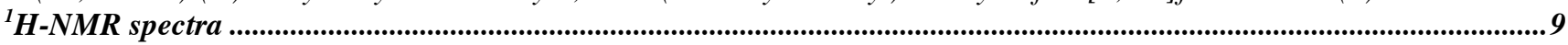

(2S)-(-)-2-Benzyloxymethyl-2,3,3-tris(methoxycarbonyl)-1,4-dioxaspiro[4.4]nonane (32)...........................................9

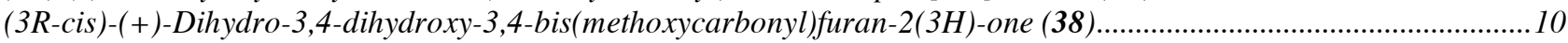

(3S-trans)-(-)-Dihydro-3,4-dihydroxy-3,4-bis(methoxycarbonyl)furan-2(3H)-one (39). ..............................................11

(2S)-2-Benzyloxymethyl-2,3-dihydroxy-3-methoxycarbonylsuccinic acid dimethyl ester (40)......................................12

(2R,3aR-cis)-(+)-Dihydro-3a,6a-bis(methoxycarbonyl)-2-phenylfuro[3,4-d][1,3]dioxol-4(3aH)-one (46)....................13

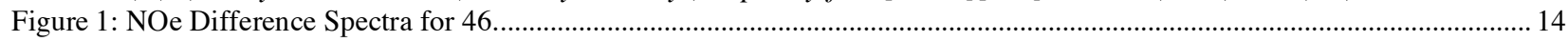

(2S,3aR-cis)-(+)-Dihydro-3a,6a-bis(methoxycarbonyl)-2-phenylfuro[3,4-d][1,3]dioxol-4(3aH)-one (47)....................15

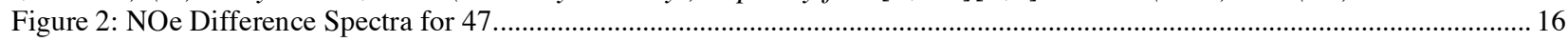

$(2 R, 3 a R, 6 a S)-(+)-4-t e r t-B u t o x y c a r b o n y l m e t h y l-3 a, 6 a-b i s(m e t h o x y c a r b o n y l)-2-$

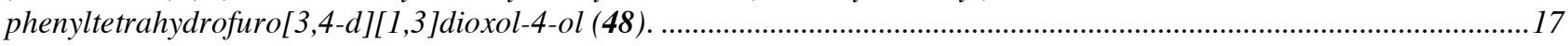

(2S,3aR,6aS)-(+)-4-tert-Butoxycarbonylmethyl-3a,6a-bis(methoxycarbonyl)-2-

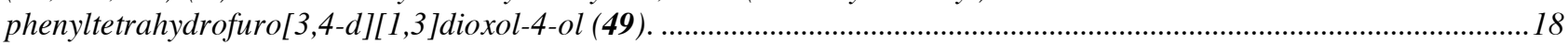

(3S,3aS-cis)-(+)-3-Hydroxy-6a-methoxy-3,3a-bis(methoxycarbonyl)tetrahydrofuro[2,3-b]furan-5-one (5).................... 19

Secondary Studies .......................................................................................................................................20

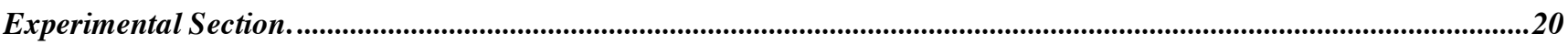

(4S,5S)-4-Benzyloxymethyl-2,2-dimethyl-4,5-bis(methoxycarbonyl) [1,3]dioxolane (12)..............................................20

(4S)-4-Benzyloxymethyl-2,2-dimethyl-4,5,5-tris(methoxycarbonyl) [1,3]dioxolane (14) ..............................................22

(3aR-cis)-Dihydro-2,2-dimethyl-3a,6a-bis(methoxycarbonyl)furo [3,4-d][1,3]dioxol-4(3aH)-one (16)........................21 
(3aR-cis)-4-tert-Butoxycarbonylmethyl-2,2-dimethyl-3a,6a-

bis(methoxycarbonyl)tetrahydrofuro[3,4-d][1,3]dioxol-4-ol (17).

(3a'R-cis)-3a',6a'-Bis(methoxycarbonyl)spiro(cyclopentane-1,2'-

dihydrofuro[3,4-d][1,3]dioxol)-4'(3a'H)-one (34).

(3aR-cis)-4-tert-Butoxycarbonylmethyl-3a,6a-

bis(methoxycarbonyl)spiro(cyclopentane-1,2'-tetrahydrofuro[3,4-d][1,3]dioxol)-4'-ol (37).

(3aR-cis)-Dihydro-3a,6a-bis(methoxycarbonyl)furo[3,4-d][1,3]dioxol-4(3aH)-one (43).

(3aR-cis)-4-tert-Butoxycarbonylmethyl-3a,6a-bis(methoxycarbonyl)tetrahydrofuro[3,4-d][1,3]dioxol-4-ol (45).

(3R-cis)-Dihydro-3,4-bis(acetoxy)-3,4-bis(methoxycarbonyl)furan-2(3H)-one

(3R-cis)-Dihydro-3,4-bis(2-bromoacetoxy)-3,4-bis(methoxycarbonyl)furan-2(3H)-one.

(3R-cis)-Dihydro-3,4-bis(methoxycarbonyl)-3,4-bis(trimethylsilanyloxy)furan-2(3H)-one ...........................................25

${ }^{1} \mathrm{H}$-NMR spectra

.26

(4S,5S)-4-Benzyloxymethyl-2,2-dimethyl-4,5-bis(methoxycarbonyl)[1,3]dioxolane (12).

Figure 2: NOESY2D Spectra for 12

(4S)-4-Benzyloxymethyl-2,2-dimethyl-4,5,5-tris(methoxycarbonyl)[1,3]dioxolane (14)....

(3aR-cis)-4-tert-Butoxycarbonylmethyl-2,2-dimethyl-3a,6a-

bis(methoxycarbonyl)tetrahydrofuro[3,4-d][1,3]dioxol-4-ol (17).

(3a'R-cis)-3a',6a'-Bis(methoxycarbonyl)spiro(cyclopentane-1,2'-

dihydrofuro [3,4-d][1,3]dioxol)-4'(3a'H)-one (34)

(3aR-cis)-4-tert-Butoxycarbonylmethyl-3a,6a-

bis(methoxycarbonyl)spiro(cyclopentane-1,2'-tetrahydrofuro[3,4-d][1,3]dioxol)-4'-ol (37)........................................34

(3aR-cis)-Dihydro-3a,6a-bis(methoxycarbonyl)furo [3,4-d][1,3]dioxol-4(3aH)-one (43) ...............................................35

(3aR-cis)-4-tert-Butoxycarbonylmethyl-3a,6a-bis(methoxycarbonyl)tetrahydrofuro[3,4-d][1,3]dioxol-4-ol (45)...........36

(3R-cis)-Dihydro-3,4-bis(methoxycarbonyl)-3,4-bis-(trimethyl-silanyloxy)furan-2(3H)-one .........................................37

(3R-cis)-Dihydro-3,4-bis(acetoxy)-3,4-bis(methoxycarbonyl)furan-2(3H)-one.........................................................38

(3R-cis)-Dihydro-3,4-bis(3-bromopropionyloxy)-3,4-bis(methoxycarbonyl)furan-2(3H)-one. 


\section{General Information}

${ }^{1} \mathrm{H}$ - and ${ }^{13} \mathrm{C}$ NMR spectra were recorded on either a Varian Gemini $400 \mathrm{MHz}$ spectrometer or a Varian Unity INOVA $500 \mathrm{MHz}$ spectrometer and chemical shifts are reported relative to tetramethylsilane as the internal standard. NOe difference and NOESY spectra were recorded on a Varian Unity INOVA $500 \mathrm{MHz}$ spectrometer. High-resolution mass spectra were obtained from the Boston College spectrometry facility. Infrared spectra were recorded using a Nicolet Avatar 360 FT-IR. Melting points were obtained on a Fisher-Johns melting point apparatus and are uncorrected. Specific rotation measurements were obtained using a Rudolph Research analytical Autopol IV automatic polarimeter. Elemental analyses were performed at Atlantic Microlab, Inc (Norcross, GA). X-ray crystallography and structure analysis for $5,16,38$, and 39 were performed at the Boston College $\mathrm{x}$-ray facility and the structural data is available as a CIF file (http://pubs.acs.org).

Dicyclopropyl ketone dimethyl acetal, ${ }^{1} \mathbf{1 0},{ }^{2}$ and protected tartrate derivatives incorporating $\mathbf{2 1},{ }^{3} \mathbf{2 2},{ }^{4}$ $\mathbf{2 3},{ }^{5} \mathbf{2 4},{ }^{6} \mathbf{2 5}^{7} \mathbf{2 6}^{8}$ and $\mathbf{2 8}^{9}$ were prepared according to literature precedent; $\mathbf{3 1}^{10}$ was prepared according to the literature procedure for the antipode and comparison of the observed specific rotation, $[\alpha]^{20}$ $+34.7^{\circ}\left(\mathrm{c} 2.90, \mathrm{CHCl}_{3}\right.$ ), with literature data for the antipode, $[\alpha]^{20}-34.0^{\circ}$ (c 2.80, $\mathrm{CHCl}_{3}$ ), was used to confirm synthesis of the desired stereoisomer. Tetrahydrofuran (THF), diethyl ether $\left(\mathrm{Et}_{2} \mathrm{O}\right)$, dichloromethane (DCM), pentane and toluene were dried by passage through a column of activated alumina under a positive pressure of nitrogen. ${ }^{11}$ Diisopropylamine (DIPA) was purchased from Aldrich and distilled from calcium hydride prior to use. Hexamethylphosphoramide (HMPA) was purchased from Aldrich, distilled from calcium hydride at $0.1 \mathrm{~mm} \mathrm{Hg}$ and stored over $4 \AA$ molecular sieves at -26 ${ }^{\circ} \mathrm{C}$ under an atmosphere of argon. Technical grade benzyloxymethyl chloride (BOM-Cl) was purchased from Fluka, fractionally distilled ${ }^{12}$ and stored at $-26{ }^{\circ} \mathrm{C}$ under an atmosphere of argon. Benzene and methanol $(\mathrm{MeOH})$ were purchased anhydrous from Acros and used as supplied. $t$-Butyl acetate was purchased from Aldrich, dried over $4 \AA$ molecular sieves and stored at ambient temperature under an atmosphere of argon. All other reagents were obtained from either Aldrich or Acros and used without further purification. Unless otherwise noted, all reactions were carried out in flame-dried glassware under an atmosphere of argon or nitrogen. The following are used throughout this section: lithium diisopropylamide (LDA), ethyl acetate (EtOAc), magnesium sulfate $\left(\mathrm{MgSO}_{4}\right)$, ammonium chloride $\left(\mathrm{NH}_{4} \mathrm{Cl}\right)$, 10-camphorsulfonic acid (CSA), trifluoroacetic acid (TFA), sodium bicarbonate $\left(\mathrm{NaHCO}_{3}\right)$ and titanium tetrachloride $\left(\mathrm{TiCl}_{4}\right)$.

\footnotetext{
${ }_{2}^{1}$ Leighton, J. L. Ph.D. Thesis, Harvard University, Cambridge, MA, 1994, p122.

2 (a) Kim, B. M.; Bae, S. J.; So, M. S.; Yoo, H. T.; Chang, S. K.; Lee, J. H.; Kang, J. Org. Lett 2001, 3, 2349-2351. (b) Mash, E. A.; Nelson, K. A.; Van Deusen, S.; Hemperly, S. B. Org. Synth 1990, 68, 92-103.

(a) For protection of tartrate with 21 see Fujita, K.; Nakai, H.; Kobayashi, S.; Inove, K.; Nojima, S.; Ohno, M. Tetrahedron Lett. 1982, 23, 3507-3510. (b) For examples of non-hydrolytic cleavage of benzylidene acetals see Greene, T. W.; Wuts, P. G. M. Protective Groups in Organic Synthesis; John Wiley \& Sons, Inc.: New York, 1999; chapter 2, 219-222.

${ }^{4}$ For protection of tartrate with 22 see (a) Narasaka, K.; Iwasawa, N.; Inoue, M.; Yamada, T; Nakashima, M.; Sugimori, J. J. Am. Chem. Soc. 1989, 111, 5340-5345. (b) Seebach, D.; Dahinden, R. E. M.; Beck, A. K.; Plattner, D. A.; Kuhnle, F. N. M. J. Org. Chem. 1995, 60, 1788-1799. (c) For an example of reductive cleavage of 1-phenylethylidene ketals see Richter, W. J. J. Org. Chem. 1981, 46, 5119-5124.

For protection of tartrate with 23 see (a) Weber, E.; Dorpinghaus, N.; Goldberg, I. J. Chem. Soc., Chem. Commun. 1988, 1566-1568. (b) Irurve, J.; Alonso-Alija, C. A.; Piniella, J. F.; Alvarez-Larena, A. Tetrahedron Asymm. 1992, 3, 1591-1596. (c) For an example of the reductive cleavage of benzophenone ketals see Mori, Y.; Asai, M.; Okumura, A.; Furukawa, H. Tetrahedron 1995, 51, 5299-5314.

${ }^{6}$ For protection of tartrate with 24 see (a) Weber, E.; Dorpinghaus, N.; Goldberg, I. J. Chem. Soc., Chem. Commun. 1988, 1566-1568. (b) Seebach, D.; Dahinden, R. E. M.; Beck, A. K.; Plattner, D. A.; Kuhnle, F. N. M. J. Org. Chem. 1995, 60, 1788-1799.

(a) For protection of tartrate with 25 see Seebach, D.; Beck, A. K.; Imwinkelried, R.; Roggo, S.; Wonnacott, A. Helv. Chim. Acta 1987, 70, 954-974. (b) For an example of oxidative cleavage of 1-t-butylmethylidene acetals see Rychnovsky, S. D.; Powell, N. A. J. Org. Chem. 1997, 62, 6460-6461.

(a) For protection of tartrate with 26 see Seebach, D.; Plattner, D. A.; Beck. A. K.; Wang, Y. M.; Hunziker, D.; Petter, W. Helv. Chim. Acta 1992, 75, 2171-2209. (b) Guindon, Y.; Ogilvie, W. W.; Bordeleau, J.; Cui, W. L.; Durkin, K.; Gorys, V.; Juteau, H.; Lemieux, R.; Liotta, D.; Simoneau, B.; Yoakim, C. J. Am. Chem. Soc. 2003, 125, 428-436. (b) For an example of oxidative cleavage of ethylidene acetals see Stork, G.; Rychnovsky, S. D. J. Am. Chem. Soc. 1987, 109, 1565-1567.

${ }_{9}^{9}$ For protection of tartrate with 28 see (a) Zheng, S.; Sogah, D. Y. Tetrahedron 1997, 53, 15469-15485. (b) Crich, D.; Hao, X. J. Org. Chem. 1999, 64, $4016-4024$.

${ }_{11}^{10}$ Crich, D.; Hao, X. J. Org. Chem. 1999, 64, 4016-4024.

${ }_{11}$ Pangborn, A. B.; Giardello, M. A.; Grubbs, R. H.; Rosen, R. K.; Timmers, F. J. Organometallics 1996, 15, 1518-1520.

${ }^{12}$ Connor, D. S.; Klein, G. W.; Taylor, G. N.; Boeckman, R. K. Jr.; Medwid, J. B. Org. Synth. 1988, VI, 101-103.
} 


\section{$\underline{\text { Main Route }}$}

\section{Experimental Section.}

(2S)-(-)-2-Benzyloxymethyl-2,3,3-tris(methoxycarbonyl)-1,4-dioxaspiro[4.4]nonane (32).

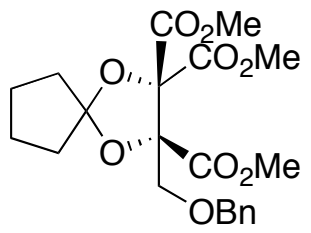

LDA, prepared by addition (over $5 \mathrm{~min}$ ) of a $2.50 \mathrm{M}$ solution of $n$-butyllithium in hexanes (7.60 $\mathrm{mL}, 19.0 \mathrm{mmol})$ to a solution of DIPA $(3.80 \mathrm{~mL}, 27.1 \mathrm{mmol})$ in THF $(60 \mathrm{~mL})$ at $-78{ }^{\circ} \mathrm{C}$, was added dropwise over $10 \mathrm{~min}$ via cannula to a solution of $\mathbf{3 1}^{13}(6.54 \mathrm{~g}, 17.9 \mathrm{mmol})$ in THF $(60 \mathrm{~mL})$ at $-78{ }^{\circ} \mathrm{C}$. After $0.5 \mathrm{~h}$, methyl chloroformate $(1.80 \mathrm{~mL}, 23.3 \mathrm{mmol})$ was added. The reaction was stirred at $-78{ }^{\circ} \mathrm{C}$ for $45 \mathrm{~min}$ and additional methyl chloroformate $(1.80 \mathrm{~mL}, 23.3 \mathrm{mmol})$ was then added. The reaction was quenched after two additional hours by addition of saturated $\mathrm{NH}_{4} \mathrm{Cl}(30 \mathrm{~mL})$. The resulting mixture was extracted with EtOAc $(3 \times 100 \mathrm{~mL})$. The organic extracts were combined, dried over $\mathrm{MgSO}_{4}$ and evaporated to yield a yellow oil $(8.7 \mathrm{~g})$. The crude product was purified by flash chromatography using silica gel (Column, 7 x $16 \mathrm{~cm}$ ) with 4:1 hexanes:EtOAc as the eluent to yield a yellow oil $(6.23 \mathrm{~g}, 66 \%)$. ${ }^{1} \mathrm{H}$ NMR $\left(400 \mathrm{MHz}, \mathrm{CDCl}_{3}, \delta\right): 1.73-1.76(\mathrm{~m}, 4 \mathrm{H}), 1.87-1.96(\mathrm{~m}, 1 \mathrm{H}), 1.97-2.02(\mathrm{~m}, 2 \mathrm{H}), 2.05-2.13$ $(\mathrm{m}, 1 \mathrm{H}), 3.71(\mathrm{~s}, 3 \mathrm{H}), 3.77(\mathrm{~s}, 3 \mathrm{H}), 3.78(\mathrm{~s}, 3 \mathrm{H}), 3.89(\mathrm{~d}, J=10.4 \mathrm{~Hz}, 1 \mathrm{H}), 4.00(\mathrm{~d}, J=10.4 \mathrm{~Hz}, 1 \mathrm{H})$, $4.55(\mathrm{~s}, 2 \mathrm{H}), 7.25-7.34(\mathrm{~m}, 5 \mathrm{H}) .{ }^{13} \mathrm{C}$ NMR (100 MHz, $\left.\mathrm{CDCl}_{3}, \delta\right): 23.6(1 \mathrm{C}), 23.8(1 \mathrm{C}), 37.7(1 \mathrm{C}), 38.5$ (1C), 52.7 (1C), 53.15 (1C), 53.3 (1C), 70.7 (1C), 73.7 (1C), 87.0 (1C), 88.3 (1C), 123.6 (1C), 127.3 (2C), 127.4 (1C), 128.1 (2C), 137.4 (1C), 165.8 (1C), 166.4 (1C), 168.7 (1C). I.R.: (NaCl, thin film) $\left(\mathrm{cm}^{-1}\right)$ : 3062 (m), 3031 (s), 2955 (vs), 2873 (s), 1759 (vs), 1627 (vw), 1495 (m), 1463 (vs), 1432 (vs). $[\alpha]^{20}-5.1^{\circ}\left(\mathrm{c} 4.79, \mathrm{CHCl}_{3}\right)$. HRMS $\mathrm{m} / z:[\mathrm{M}+\mathrm{Na}]^{+}$calcd for $\mathrm{C}_{21} \mathrm{H}_{26} \mathrm{O}_{9} \mathrm{Na}, 445.1475$; found, 445.1481.

(3R-cis)-(+)-Dihydro-3,4-dihydroxy-3,4-bis(methoxycarbonyl)furan-2(3H)-one (38).

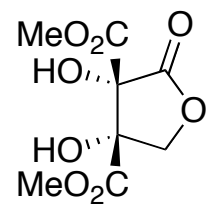

Triester 32 (3.28 g, $7.77 \mathrm{mmol})$ was dissolved in a 20:10:1 (v/v/v) mixture of DCM:TFA: $\mathrm{H}_{2} \mathrm{O}$ $(38.75 \mathrm{~mL})$, the reaction flask was sealed and the mixture was heated at $65{ }^{\circ} \mathrm{C}$ for 24 hours. After cooling to ambient temperature, the solution was diluted with toluene $(50 \mathrm{~mL})$ and evaporated. ${ }^{14}$ The crude product was purified by flash chromatography using silica gel (Column, $5 \mathrm{x} 16 \mathrm{~cm}$ ) with a solvent gradient of 9:1 DCM:EtOAc to 4:1 DCM:EtOAc as the eluent to yield $\mathbf{3 8}$ as a white powder $(0.900 \mathrm{~g}$, $50 \%), 39$ as a pale yellow clear oil which solidifies on standing $(0.180 \mathrm{~g}, 10 \%)$ and $\mathbf{4 0}$ as a yellow oil, which was never obtained completely pure $(0.163 \mathrm{~g}, 9 \%)$. 38: $\mathrm{Mp} 122-123{ }^{\circ} \mathrm{C} .{ }^{1} \mathrm{H}$ NMR $(400 \mathrm{MHz}$, $\left.\mathrm{CDCl}_{3}, \delta\right): 3.81(\mathrm{~s}, 3 \mathrm{H}) 3.91(\mathrm{~s}, 3 \mathrm{H}), 4.11(\mathrm{~s}, 2 \mathrm{H}), 4.47(\mathrm{~d}, J=10.4 \mathrm{~Hz}, 1 \mathrm{H}), 4.86(\mathrm{~d}, J=10.4 \mathrm{~Hz}, 1 \mathrm{H})$. ${ }^{13} \mathrm{C}$ NMR (100 MHz, $\left.\mathrm{CDCl}_{3}, \delta\right): 53.5$ (1C), 54.6 (1C), 72.4 (1C), 79.7 (1C), 79.8 (1C), 166.8 (1C), 168.0 (1C), 169.0 (1C). I.R.: (KBr) $\left(\mathrm{cm}^{-1}\right)$ : 3471 (s), 3371 (s), 2968 (w), 1791 (s), 1759 (s), 1741 (s), $1476(\mathrm{~m}), 1445(\mathrm{~m}) \cdot[\alpha]^{20}{ }_{\mathrm{D}}+68.0^{\circ}\left(\mathrm{c} 1.10, \mathrm{CHCl}_{3}\right)$. HRMS m/z: $[\mathrm{M}+\mathrm{Na}]^{+}$calcd for $\mathrm{C}_{8} \mathrm{H}_{10} \mathrm{O}_{8} \mathrm{Na}$, 257.0273; found, 257.0271 .

\footnotetext{
${ }_{14}^{13}$ See General Information section.

${ }^{14}$ Evans, D. A.; Trotter, B. W.; Barrow, J. C. Tetrahedron 1997, 53, 8779-8794.
} 


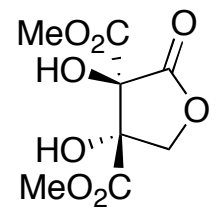

Mp 120-121 ${ }^{\circ} \mathrm{C} .{ }^{1} \mathrm{H}$ NMR (400 MHz, $\left.\mathrm{CDCl}_{3}, \delta\right): 3.91$ (s, 3H), 3.95 (s, 3H), 4.42 (d, J = 9.6 Hz, $1 \mathrm{H}), 4.51(\mathrm{~d}, J=9.6 \mathrm{~Hz}, 1 \mathrm{H}) .{ }^{13} \mathrm{C} \mathrm{NMR}\left(100 \mathrm{MHz}, \mathrm{CDCl}_{3}\right): 54.3$ (1C), 54.6 (1C), 69.9 (1C), 80.9 (1C), 81.0 (1C), 166.5 (1C), 169.3 (1C), 170.3 (1C). I.R.: (KBr) $\left(\mathrm{cm}^{-1}\right): 3517(\mathrm{~m}), 3451(\mathrm{~s}), 3019$ (w), 2992 (w), $2965(w), 1797(s), 1785(\mathrm{~s}), 1751(\mathrm{~s}) .[\alpha]^{20}{ }_{\mathrm{D}}-105.7^{\circ}$ (c 1.18, $\mathrm{CHCl}_{3}$ ). Anal. Calcd for $\mathrm{C}_{8} \mathrm{H}_{10} \mathrm{O}_{8}$ : C, 41.03; H, 4.30. Found: $\mathrm{C}, 41.18 ; \mathrm{H}, 4.35$. HRMS $\mathrm{m} / z:[\mathrm{M}+\mathrm{Na}]^{+}$calcd for $\mathrm{C}_{8} \mathrm{H}_{10} \mathrm{O}_{8} \mathrm{Na}, 257.0273$; found, 257.0278 .

(2S)-2-Benzyloxymethyl-2,3-dihydroxy-3-methoxycarbonylsuccinic acid dimethyl ester (40).

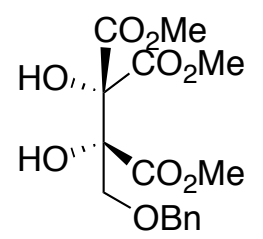

${ }^{1} \mathrm{H}$ NMR $\left(400 \mathrm{MHz}, \mathrm{CDCl}_{3}, \delta\right): 3.78(\mathrm{~s}, 3 \mathrm{H}), 3.79(\mathrm{~s}, 3 \mathrm{H}), 3.82(\mathrm{~s}, 3 \mathrm{H}), 4.02(\mathrm{~d}, J=10 \mathrm{~Hz}, 1 \mathrm{H})$, $4.17(\mathrm{~d}, J=10 \mathrm{~Hz}, 1 \mathrm{H}), 4.32(\mathrm{~s}, 2 \mathrm{H}), 4.53(\mathrm{~d}, J=12 \mathrm{~Hz}, 1 \mathrm{H}), 4.62(\mathrm{~d}, J=12 \mathrm{~Hz}, 1 \mathrm{H}), 7.17-7.35(\mathrm{~m}$, 5H). ). ${ }^{13} \mathrm{C} \mathrm{NMR}\left(100 \mathrm{MHz}, \mathrm{CDCl}_{3}, \delta\right): 53.3$ (1C), 53.7 (1C), 71.3 (1C), 73.9 (2C), 81.1 (1C), 81.4 (1C), 127.58 (2C), 127.64 (1C), 128.2 (2C), 137.4 (1C), 167.9 (1C), 168.1 (1C), 171.2 (1C). HRMS $\mathrm{m} / \mathrm{z}:[\mathrm{M}+\mathrm{Na}]^{+}$calcd for $\mathrm{C}_{16} \mathrm{H}_{20} \mathrm{O}_{9} \mathrm{Na}, 379.1005$; found, 379.1002 .

(2R,3aR-cis)-(+)-Dihydro-3a,6a-bis(methoxycarbonyl)-2-phenylfuro[3,4-d][1,3]dioxol-4(3aH)-one (46).<smiles>CO[C@H](c1ccccc1)[C@@]1(C)COC(=O)[C@]1(C)OC</smiles>

Lactone 38 (1.30 g, $5.55 \mathrm{mmol})$ and p-toluenesulfonic acid $(0.200 \mathrm{~g}, 1.05 \mathrm{mmol})$ were dissolved in a mixture of toluene $(60 \mathrm{~mL})$ and benzaldehyde $(1.70 \mathrm{~mL}, 16.7 \mathrm{mmol})$. The reaction was heated at reflux and stirred for 48 hours with excess water being removed from the reaction via azeotropic distillation into a Dean-Stark apparatus filled with $4 \AA$ molecular sieves activated by flame-drying under vacuum (in order to nudge the reaction to completion it is necessary to drain the Dean-Stark apparatus periodically and reactivate or replace the molecular sieves). After cooling to ambient temperature, the reaction mixture was washed through a plug of silica gel with EtOAc $(3 \mathrm{x} 75 \mathrm{~mL})$ and the filtrate was evaporated to yield a 2:1 mixture of stereoisomers as a clear oil (1.8 g). Flash chromatography using silica gel (Column, $5 \times 16 \mathrm{~cm})$ with 3:1 hexanes:EtOAc as the eluent allows clean separation of $46(0.54$ $\mathrm{g}, 30 \%)$ and $47(1.08 \mathrm{~g}, 60 \%)$. ${ }^{1} \mathrm{H}$ NMR $\left(400 \mathrm{MHz}, \mathrm{CDCl}_{3}, \delta\right): 3.78(\mathrm{~s}, 3 \mathrm{H}), 3.87(\mathrm{~s}, 3 \mathrm{H}), 4.68(\mathrm{~d}, J=$ $10.8 \mathrm{~Hz}, 1 \mathrm{H}), 4.99(\mathrm{~d}, J=10.8 \mathrm{~Hz}, 1 \mathrm{H}), 6.11(\mathrm{~s}, 1 \mathrm{H}), 7.35-7.45(\mathrm{~m}, 3 \mathrm{H}), 7.50-7.60(\mathrm{~m}, 2 \mathrm{H}) .{ }^{13} \mathrm{C}$ NMR (100 MHz, $\left.\mathrm{CDCl}_{3}, \delta\right): 53.6$ (1C), 53.8 (1C), 72.6 (1C), 87.1 (1C), 87.4 (1C), 107.5 (1C), 127.2 (2C), 128.4 (2C), 130.5 (1C), 133.5 (1C), 162.7 (1C), 166.2 (1C), 168.4 (1C). I.R.: (NaCl, thin film) ( $\left.\mathrm{cm}^{-1}\right)$ : 3043 (w), 3012 (w), 2955 (m), 2905 (w), 1791 (s), 1772 (s), 1746 (s), 1627 (w), 1470 (m), 1438 (m), 
$1407(\mathrm{~m}) \cdot[\alpha]^{20}{ }_{\mathrm{D}}+91.4^{\circ}\left(\mathrm{c} 1.47, \mathrm{CHCl}_{3}\right)$. HRMS m/z: $[\mathrm{M}+\mathrm{Na}]^{+}$calcd for $\mathrm{C}_{15} \mathrm{H}_{14} \mathrm{O}_{8} \mathrm{Na}, 345.0586$; found, 345.0575 .

(2S,3aR-cis)-(+)-Dihydro-3a,6a-bis(methoxycarbonyl)-2-phenylfuro[3,4-d][1,3]dioxol-4(3aH)-one (47).<smiles>COC(=O)[C@@]1(C)C(=O)OC[C@@]1(C)C(OC)OC</smiles>

${ }^{1} \mathrm{H}$ NMR $\left(400 \mathrm{MHz}, \mathrm{CDCl}_{3}, \delta\right): 3.86(\mathrm{~s}, 3 \mathrm{H}), 3.87(\mathrm{~s}, 3 \mathrm{H}), 4.61(\mathrm{~d}, J=10.4 \mathrm{~Hz}, 1 \mathrm{H}), 4.88(\mathrm{~d}, J=$ $10.4 \mathrm{~Hz}, 1 \mathrm{H}), 6.54(\mathrm{~s}, 1 \mathrm{H}), 7.35-7.50(\mathrm{~m}, 5 \mathrm{H}) .{ }^{13} \mathrm{C}$ NMR $\left(100 \mathrm{MHz}, \mathrm{CDCl}_{3}, \delta\right): 55.7(1 \mathrm{C}), 55.8(1 \mathrm{C})$, 70.1 (1C), 86.9 (1C), 90.0 (1C), 110.1 (1C), 126.8 (2C), 128.4 (2C), 130.4 (1C), 133.6 (1C), 164.3 (1C), 166.1 (1C), 168.3 (1C). I.R.: (NaCl, thin film) $\left(\mathrm{cm}^{-1}\right): 3037(\mathrm{w}), 2962(\mathrm{~m}), 2848(\mathrm{w}), 1803(\mathrm{~s}), 1766$ (s), $1747(\mathrm{~s}), 1469(\mathrm{~m}), 1451(\mathrm{~m}), 1401(\mathrm{~m}) \cdot[\alpha]_{\mathrm{D}}^{20}+109.5^{\circ}\left(\mathrm{c} 1.41, \mathrm{CHCl}_{3}\right) \cdot \mathrm{HRMS} \mathrm{m} / \mathrm{z}:\left[\mathrm{M}+\mathrm{Na}^{+} \mathrm{calcd}\right.$ for $\mathrm{C}_{15} \mathrm{H}_{14} \mathrm{O}_{8} \mathrm{Na}$, 345.0586; found, 345.0571.

(2R,3aR,6aS)-(+)-4-tert-Butoxycarbonylmethyl-3a,6a-bis(methoxycarbonyl)-2phenyltetrahydrofuro[3,4-d][1,3]dioxol-4-ol (48).<smiles>CCOC(=O)C[C@@]1(O)OC[C@](C)([C@H](OC)c2ccccc2)[C@@]1(C)OC</smiles>

A $2.50 \mathrm{M}$ solution of $n$-butyllithium in hexanes $(1.2 \mathrm{~mL}, 3.0 \mathrm{mmol}$ ) was added dropwise (over 5 $\min )$ to a solution of DIPA $(0.51 \mathrm{~mL}, 3.6 \mathrm{mmol})$ in $\mathrm{Et}_{2} \mathrm{O}(8 \mathrm{~mL})$ at $-78{ }^{\circ} \mathrm{C}$. After $30 \mathrm{~min}, t$-butyl acetate $(0.61 \mathrm{~mL}, 4.5 \mathrm{mmol})$ was added dropwise over one minute and the reaction was stirred at $-78{ }^{\circ} \mathrm{C}$ for 40 min, then warmed to $-40{ }^{\circ} \mathrm{C}$. In a second flask, $46(0.180 \mathrm{~g}, 0.55 \mathrm{mmol})$ was dissolved in $\mathrm{Et}_{2} \mathrm{O}(20 \mathrm{~mL})$ and $\mathrm{TiCl}_{4}(0.55 \mathrm{~mL}, 1.0 \mathrm{M}$ in DCM, $0.55 \mathrm{mmol})$ was added in one portion. The resulting yellow homogeneous solution was cooled to $-40{ }^{\circ} \mathrm{C}$ and part of the lithium $t$-butyl acetate stock solution (3.67 $\mathrm{mL}, 0.30 \mathrm{M}, 1.1 \mathrm{mmol}$ ) was added dropwise over $2 \mathrm{~min}$. After $3.5 \mathrm{~h}$, the reaction was quenched by addition of a saturated solution of $\mathrm{NH}_{4} \mathrm{Cl}(10 \mathrm{~mL})$ and the aqueous layer was extracted with $\mathrm{Et}_{2} \mathrm{O}(2 \times 30$ $\mathrm{mL}$ ). The organic extracts were combined, dried over $\mathrm{MgSO}_{4}$ and evaporated to yield a yellow oil $(0.270 \mathrm{~g})$. The crude product was purified by flash chromatography using silica gel (Column, $3 \times 16$ $\mathrm{cm})$ with $1 \%$ EtOAc in DCM as the eluent to yield $48(0.148 \mathrm{~g}, 62 \%)$. ${ }^{1} \mathrm{H} \mathrm{NMR}\left(400 \mathrm{MHz}, \mathrm{CDCl}_{3}, \delta\right)$ : $1.50(\mathrm{~s}, 9 \mathrm{H}), 2.83(\mathrm{~d}, J=16.8 \mathrm{~Hz}, 1 \mathrm{H}), 3.05(\mathrm{~d}, J=16.8 \mathrm{~Hz}, 1 \mathrm{H}), 3.81(\mathrm{~s}, 3 \mathrm{H}), 3.86(\mathrm{~s}, 3 \mathrm{H}), 4.22(\mathrm{~d}, J=$ $10.8 \mathrm{~Hz}, 1 \mathrm{H}), 4.93(\mathrm{~d}, J=10.8 \mathrm{~Hz}, 1 \mathrm{H}), 5.99(\mathrm{~s}, 1 \mathrm{H}), 6.17(\mathrm{~s}, 1 \mathrm{H}), 7.38-7.42(\mathrm{~m}, 3 \mathrm{H}), 7.50-7.61(\mathrm{~m}$, 2H). ${ }^{13} \mathrm{C} \mathrm{NMR}\left(100 \mathrm{MHz}, \mathrm{CDCl}_{3}, \delta\right): 28.1$ (3C), 38.4 (1C), 53.0 (1C), 53.2 (1C), 73.5 (1C), 82.8 (1C), 91.5 (1C), 96.1 (1C), 104.5 (1C), 107.1 (1C), 127.4 (2C), 128.3 (2C), 130.2 (1C), 134.7 (1C), 166.0 (1C), $167.6(1 \mathrm{C}), 170.0(1 \mathrm{C})$. IR (NaCl, thin film) $\left(\mathrm{cm}^{-1}\right)$ : $3377(\mathrm{br}, \mathrm{s}) 1649(\mathrm{~s}), 1688(\mathrm{~s}) .[\alpha]^{20}{ }_{\mathrm{D}}+36.7^{\circ}(\mathrm{c}$ 2.93, $\mathrm{CHCl}_{3}$ ). HRMS m/z: [M+Na] $]^{+}$calcd for $\mathrm{C}_{21} \mathrm{H}_{26} \mathrm{O}_{10} \mathrm{Na}, 461.1424$; found, 461.1426 . 
(2S,3aR,6aS)-(+)-4-tert-Butoxycarbonylmethyl-3a,6a-bis(methoxycarbonyl)-2phenyltetrahydrofuro[3,4-d][1,3]dioxol-4-ol (49)<smiles>COC(=O)C[C@@]1(O)OC[C@](C)([C@H](OC)c2ccccc2)[C@@]1(C)OC(C)(C)C</smiles>

A $2.5 \mathrm{M}$ solution of $n$-butyllithium in hexanes $(1.2 \mathrm{~mL}, 3.0 \mathrm{mmol}$ ) was added dropwise (over 5 min) to a solution of DIPA $(0.51 \mathrm{~mL}, 3.6 \mathrm{mmol})$ in $\mathrm{Et}_{2} \mathrm{O}(8 \mathrm{~mL})$ at $-78{ }^{\circ} \mathrm{C}$. After $30 \mathrm{~min}, t$-butyl acetate $(0.61 \mathrm{~mL}, 4.5 \mathrm{mmol})$ was added dropwise over one min and the reaction was stirred at $-78{ }^{\circ} \mathrm{C}$ for 40 min, then warmed to $-40{ }^{\circ} \mathrm{C}$. In a second flask, $47(0.300 \mathrm{~g}, 0.93 \mathrm{mmol})$ was dissolved in $\mathrm{Et}_{2} \mathrm{O}(35 \mathrm{~mL})$ and $\mathrm{TiCl}_{4}(0.93 \mathrm{~mL}, 1.0 \mathrm{M}$ in DCM, $0.93 \mathrm{mmol})$ was added in one portion. The resulting yellow homogeneous solution was cooled to $-40{ }^{\circ} \mathrm{C}$ and part of the lithium $t$-butyl acetate stock solution $(5.58$ $\mathrm{mL}, 0.30 \mathrm{M}, 1.7 \mathrm{mmol}$ ) was added dropwise over $2 \mathrm{~min}$. After 3.5 hours, the reaction was quenched by addition of a saturated solution of $\mathrm{NH}_{4} \mathrm{Cl}(15 \mathrm{~mL})$ and the aqueous layer was extracted with $\mathrm{Et}_{2} \mathrm{O}(2 \times 35$ $\mathrm{mL})$. The organic extracts were combined, dried over $\mathrm{MgSO}_{4}$ and evaporated to yield a yellow oil (407 $\mathrm{mg}$ ). The crude product was purified by flash chromatography using silica gel with 3:1 hexanes:EtOAc as the eluent to yield a mixture of regioisomers $(0.288 \mathrm{~g})$ resulting from attack at the lactone carbonyl and attack at one of the two ester functionalities. Chromatography using silica gel (Column, $3 \mathrm{x} 16 \mathrm{~cm}$ ) with $1 \%$ EtOAc in DCM cleanly separated this mixture yielding 49 as a clear oil $(0.166 \mathrm{~g}, 41 \%) .{ }^{1} \mathrm{H}$ $\operatorname{NMR}\left(400 \mathrm{MHz}, \mathrm{CDCl}_{3}, \delta\right): 1.47(\mathrm{~s}, 9 \mathrm{H}), 2.87(\mathrm{~d}, J=17.2 \mathrm{~Hz}, 1 \mathrm{H}), 2.95(\mathrm{~d}, J=17.2 \mathrm{~Hz}, 1 \mathrm{H}), 3.83(\mathrm{~s}$, $3 \mathrm{H}), 3.86(\mathrm{~s}, 3 \mathrm{H}), 4.14(\mathrm{~d}, J=10.4 \mathrm{~Hz}, 1 \mathrm{H}), 4.80(\mathrm{~d}, J=10.4 \mathrm{~Hz}, 1 \mathrm{H}), 5.97(\mathrm{~s}, 1 \mathrm{H}), 6.24(\mathrm{~s}, 1 \mathrm{H}), 7.40-$ $7.44(\mathrm{~m}, 3 \mathrm{H}), 7.52-7.57(\mathrm{~m}, 2 \mathrm{H}) .{ }^{13} \mathrm{C} \mathrm{NMR}\left(100 \mathrm{MHz}, \mathrm{CDCl}_{3}, \delta\right): 28.1(3 \mathrm{C}), 38.6(1 \mathrm{C}), 52.9(1 \mathrm{C}), 53.0$ (1C), 71.9 (1C), 82.6 (1C), 92.5 (1C), 96.3 (1C), 105.0 (1C), 108.3 (1C), 127.3 (2C), 128.2 (2C), 130.1 (1C), 134.3 (1C), 167.5 (1C), 168.8 (1C), 171.0 (1C). I.R.: ( NaCl, thin film) $\left(\mathrm{cm}^{-1}\right): 3377$ (br,s) 1649 (s), $1688(\mathrm{~s}) .[\alpha]^{20}{ }_{D}+41.3^{\circ}$ (c 3.10, $\mathrm{CHCl}_{3}$ ). HRMS m/z: $[\mathrm{M}+\mathrm{Na}]^{+}$calcd for $\mathrm{C}_{21} \mathrm{H}_{26} \mathrm{O}_{10} \mathrm{Na}, 461.1424$; found, 461.1425 .

(3S,3aS-cis)-(+)-3-Hydroxy-6a-methoxy-3,3a-bis(methoxycarbonyl)tetrahydrofuro[2,3- $b]$ furan-5-one (5).

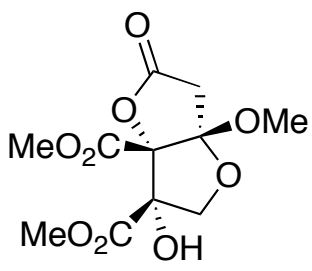

Lactol $48(0.100 \mathrm{~g}, 0.228 \mathrm{mmol})$ and $10 \% \mathrm{Pd} / \mathrm{C}(15 \mathrm{mg})$ were stirred as a suspension in anhydrous $\mathrm{MeOH}(8 \mathrm{~mL})$ under a balloon of $\mathrm{H}_{2}$ for 3 hours. Additional $10 \% \mathrm{Pd} / \mathrm{C}(15 \mathrm{mg})$ was added and the reaction was stirred in an atmosphere of $\mathrm{H}_{2}$ gas (balloon) overnight. The crude reaction mixture was then vacuum filtered through a plug of celite and the filtrate was evaporated to yield an inseparable mixture of hemiacetals $(0.079 \mathrm{~g})$. $\left\{\mathbf{5 0}:{ }^{\mathrm{H}} \mathrm{H} \mathrm{NMR}\left(400 \mathrm{MHz}, \mathrm{CDCl}_{3}, \delta\right): 1.45(\mathrm{~s}, 9 \mathrm{H}), 1.45(\mathrm{~s}, 9 \mathrm{H}), 2.706\right.$ $(\mathrm{s}, 1 \mathrm{H}), 2.710(\mathrm{~s}, 1 \mathrm{H}), 2.81(\mathrm{~s}, 1 \mathrm{H}), 2.82(\mathrm{~s}, 1 \mathrm{H}), 3.740(\mathrm{~s}, 3 \mathrm{H}), 3.741(\mathrm{~s}, 3 \mathrm{H}), 3.78(\mathrm{~s}, 3 \mathrm{H}), 3.82(\mathrm{~s}, 3 \mathrm{H})$ $4.05(\mathrm{~d}, J=10 \mathrm{~Hz}, 1 \mathrm{H}), 4.20(\mathrm{~d}, J=10 \mathrm{~Hz}, 1 \mathrm{H}), 4.65(\mathrm{~d}, J=5.2 \mathrm{~Hz}, 1 \mathrm{H}), 4.68(\mathrm{~d}, J=5.2 \mathrm{~Hz}, 1 \mathrm{H})\}$. The crude product and CSA $(0.020 \mathrm{~g}, 0.086 \mathrm{mmol})$ were dissolved in anhydrous $\mathrm{MeOH}(15 \mathrm{~mL})$. The reaction was heated at reflux overnight. After cooling to ambient temperature, the solvent was evaporated and the crude product was purified by chromatography using silica gel (Column, $3 \mathrm{x} 16 \mathrm{~cm}$ ) with 1:2 hexanes:EtOAc as the eluent to yield a white solid (0.040 g, 63\% over two steps). X-ray quality crystals were grown from a mixture of DCM and hexanes. Mp 118-120 ${ }^{\circ} \mathrm{C} .{ }^{1} \mathrm{H}$ NMR (400 
$\left.\mathrm{MHz}, \mathrm{CDCl}_{3}\right):$ 2.91-2.98 (apparent ABq, 2H), 3.29 (s, 3H), $3.77(\mathrm{~s}, 6 \mathrm{H}), 4.17(\mathrm{~d}, J=10.4 \mathrm{~Hz}, 1 \mathrm{H}), 4.54$ $(\mathrm{d}, J=10.4 \mathrm{~Hz}, 1 \mathrm{H}) .{ }^{13} \mathrm{C} \mathrm{NMR}\left(100 \mathrm{MHz}, \mathrm{CDCl}_{3}\right): 38.1$ (1C), 51.9 (1C), 52.8 (1C), 53.2 (1C), 74.5 (1C), 82.3 (1C), 93.7 (1C), 111.8 (1C), 165.6 (1C), 168.9 (1C), 171.3 (1C). I.R.: (KBr) $\left(\mathrm{cm}^{-1}\right): 3451$ (s), $3004(\mathrm{w}), 2953(\mathrm{w}), 2930(\mathrm{w}), 1797(\mathrm{~s}), 1742(\mathrm{~s}) . \quad[\alpha]_{\mathrm{D}}^{20}+21.4^{\circ}\left(\mathrm{c} 0.64, \mathrm{CHCl}_{3}\right)$. Anal. Calcd for $\mathrm{C}_{11} \mathrm{H}_{14} \mathrm{O}_{9}$ : C, 45.52; H, 4.86. Found: C, 45.55; H, 4.95. 


\section{${ }^{1}$ H-NMR spectra}

(2S)-(-)-2-Benzyloxymethyl-2,3,3-tris(methoxycarbonyl)-1,4-dioxaspiro[4.4]nonane (32).

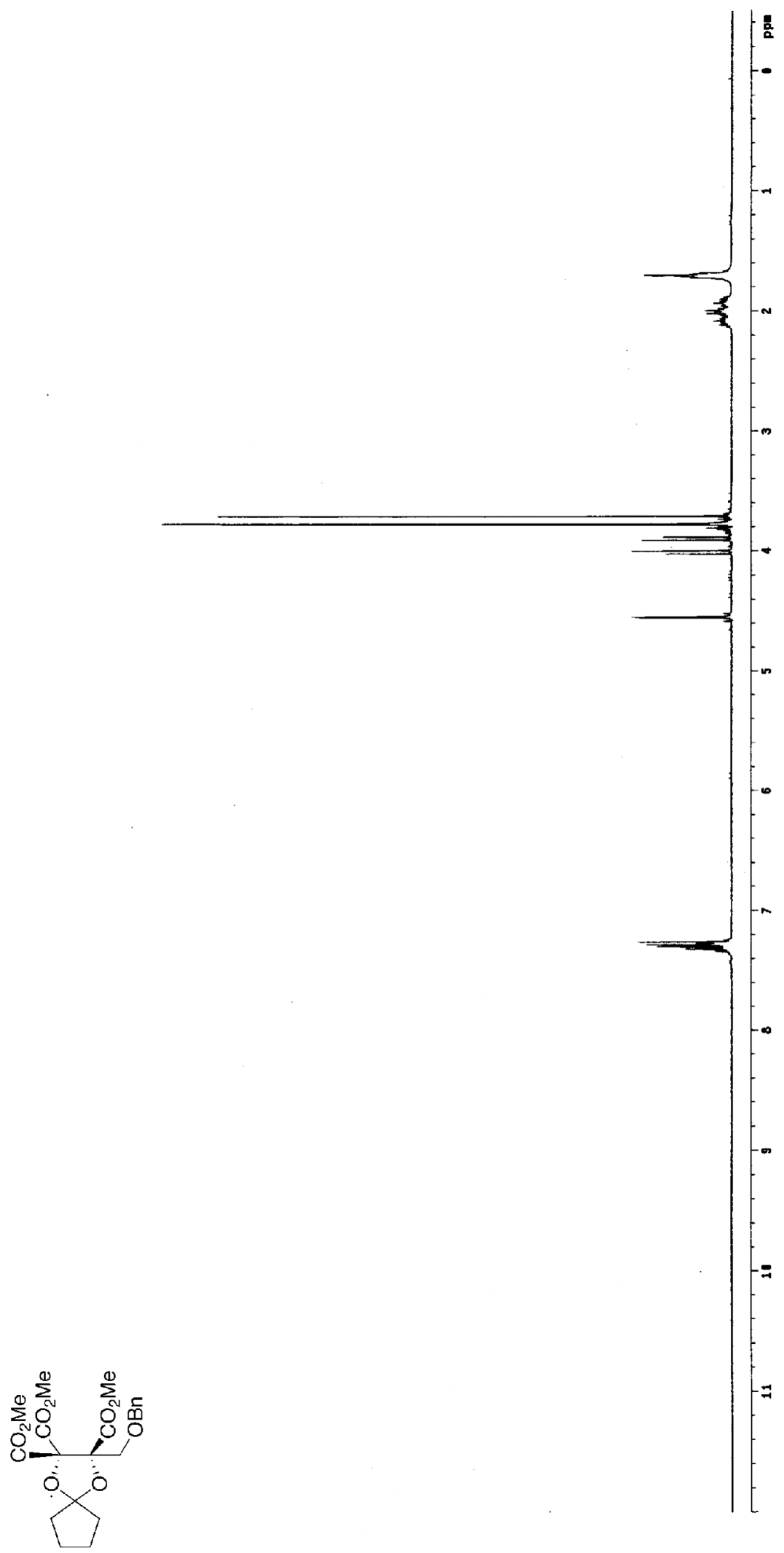


(3R-cis)-(+)-Dihydro-3,4-dihydroxy-3,4-bis(methoxycarbonyl)furan-2(3H)-one (38).
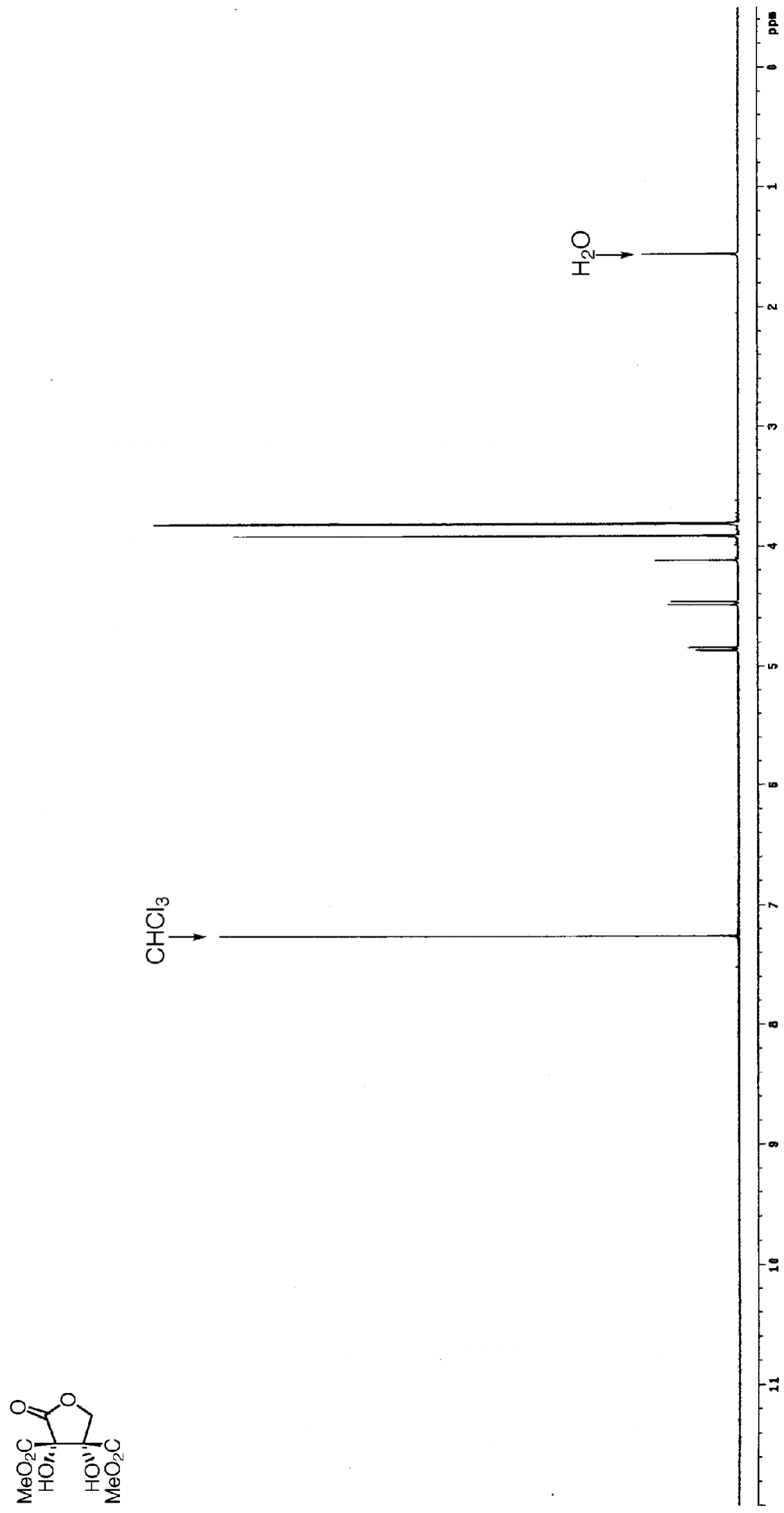
(3S-trans)-(-)-Dihydro-3,4-dihydroxy-3,4-bis(methoxycarbonyl)furan-2(3H)-one (39).

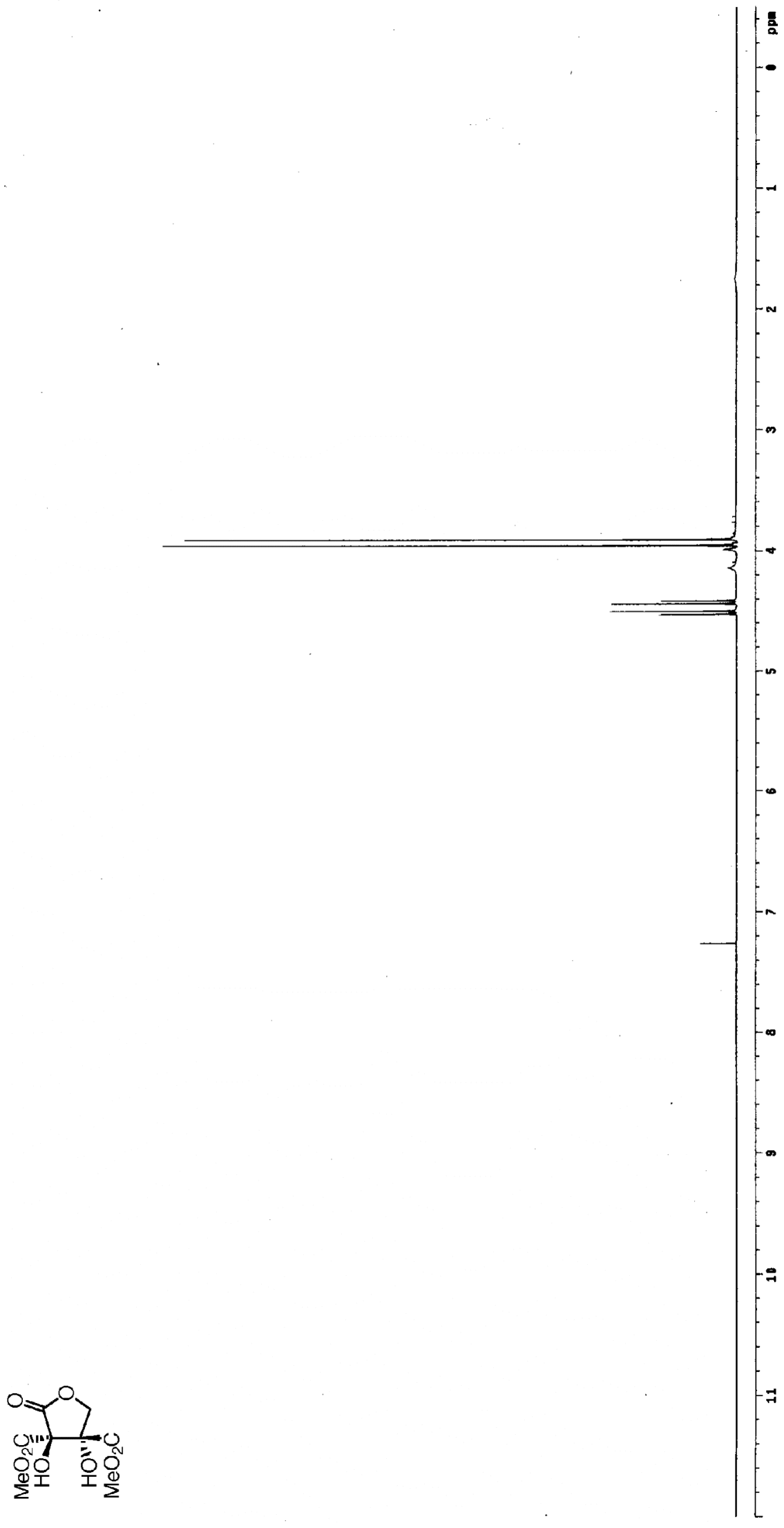


(2S)-2-Benzyloxymethyl-2,3-dihydroxy-3-methoxycarbonylsuccinic acid dimethyl ester (40).

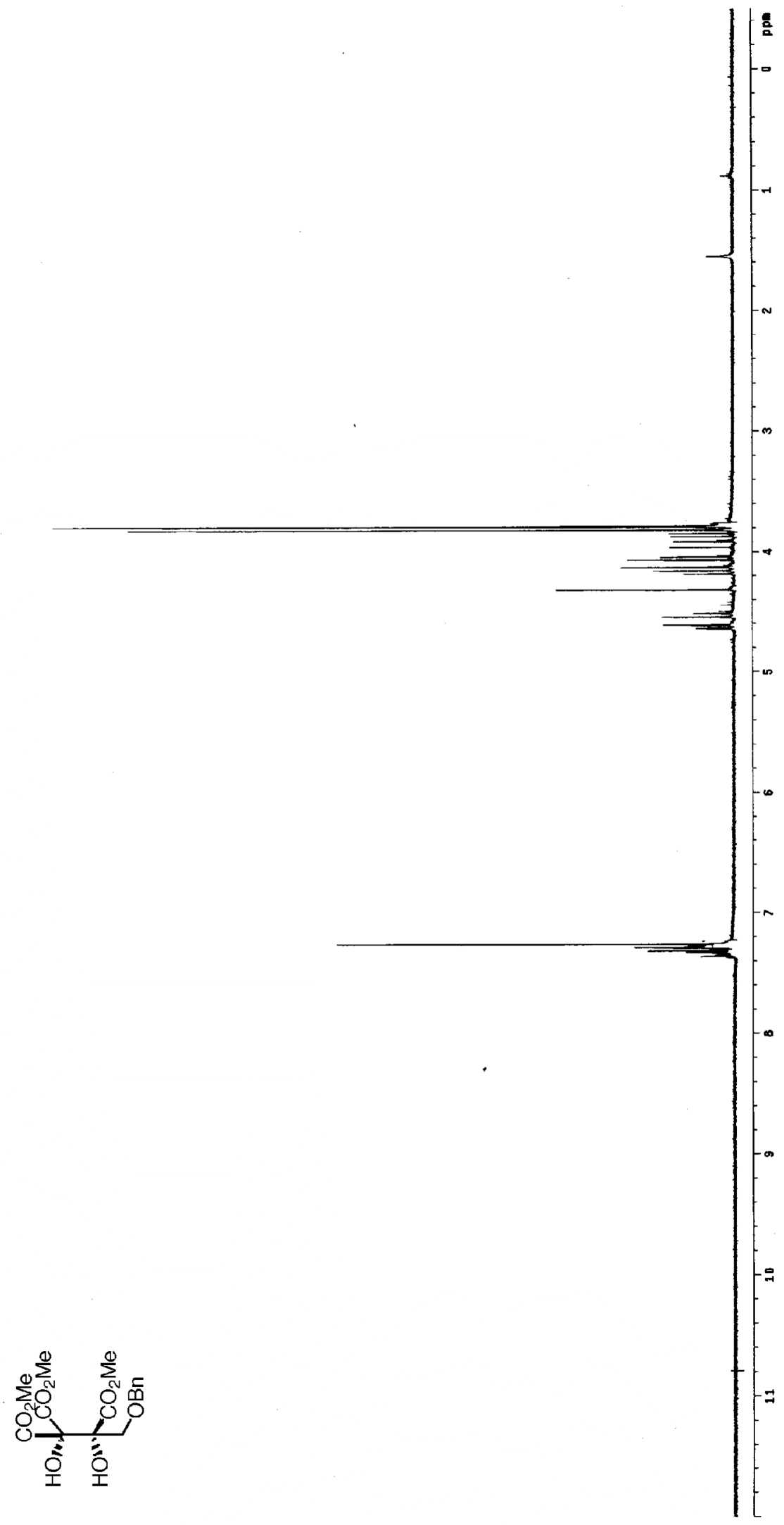


(2R,3aR-cis)-(+)-Dihydro-3a,6a-bis(methoxycarbonyl)-2-phenylfuro[3,4- $d][1,3]$ dioxol-4(3aH)-one (46).

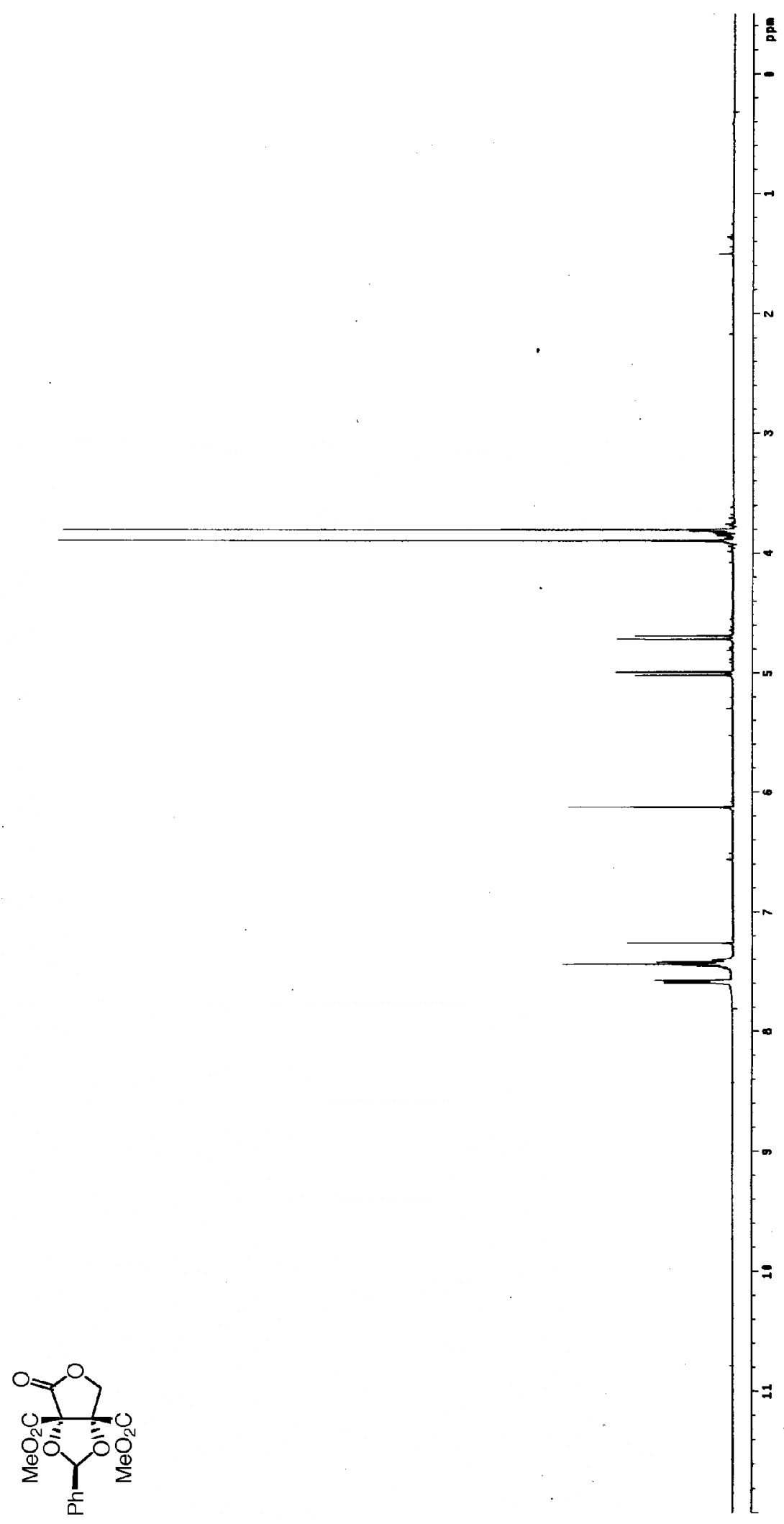




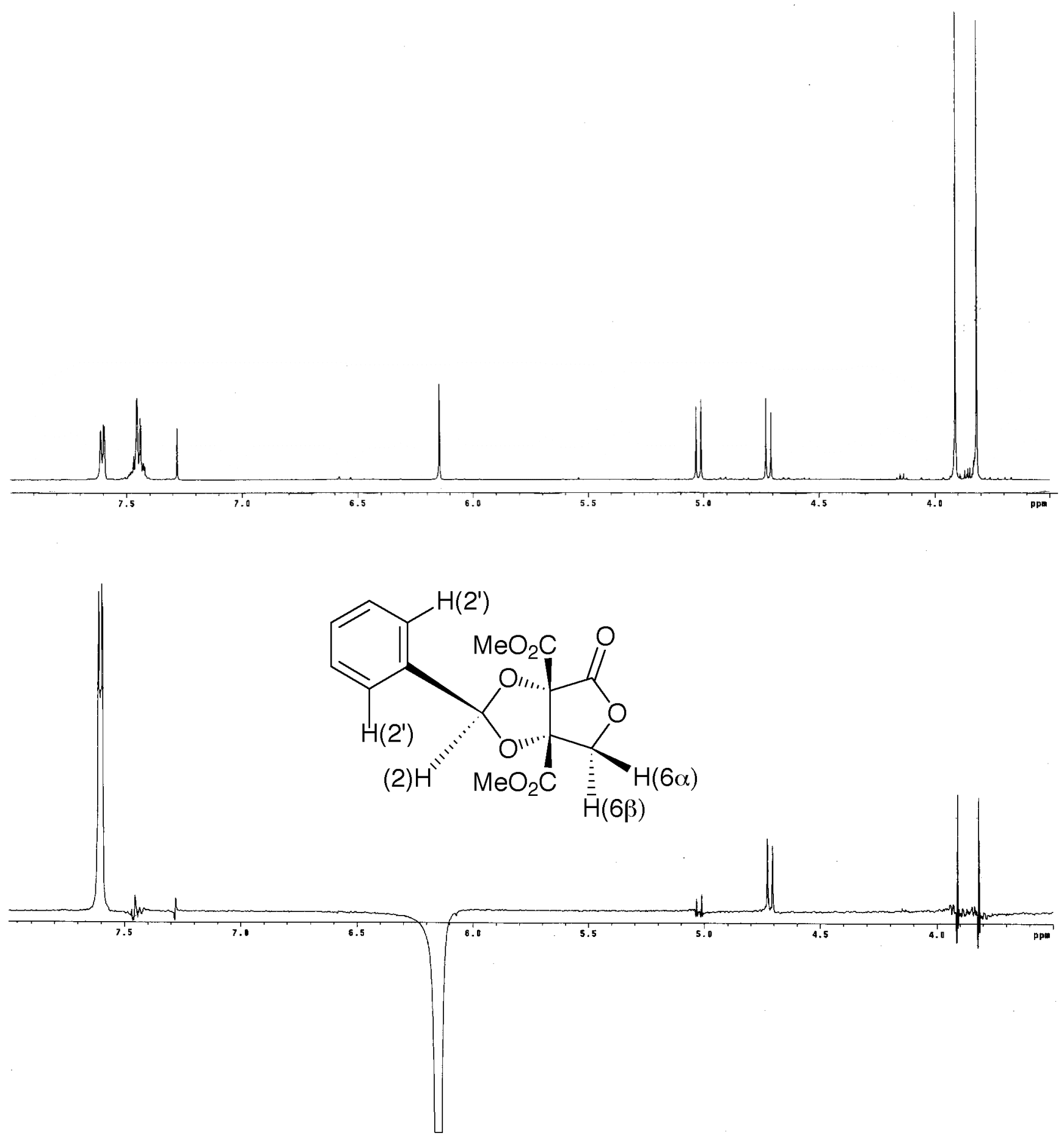

Figure 1: NOe Difference Spectra for 46.

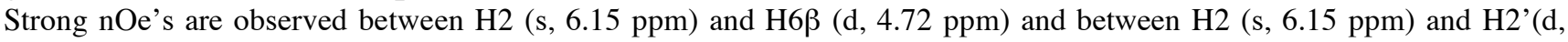
$7.60 \mathrm{ppm}$ ). The H2/H6 3 interaction is indicative of the (2R)-benzylidene stereoisomer. Compare Figure 2 (page S16). 
(2S,3aR-cis)-(+)-Dihydro-3a,6a-bis(methoxycarbonyl)-2-phenylfuro[3,4-d][1,3]dioxol-4(3aH)-one (47).

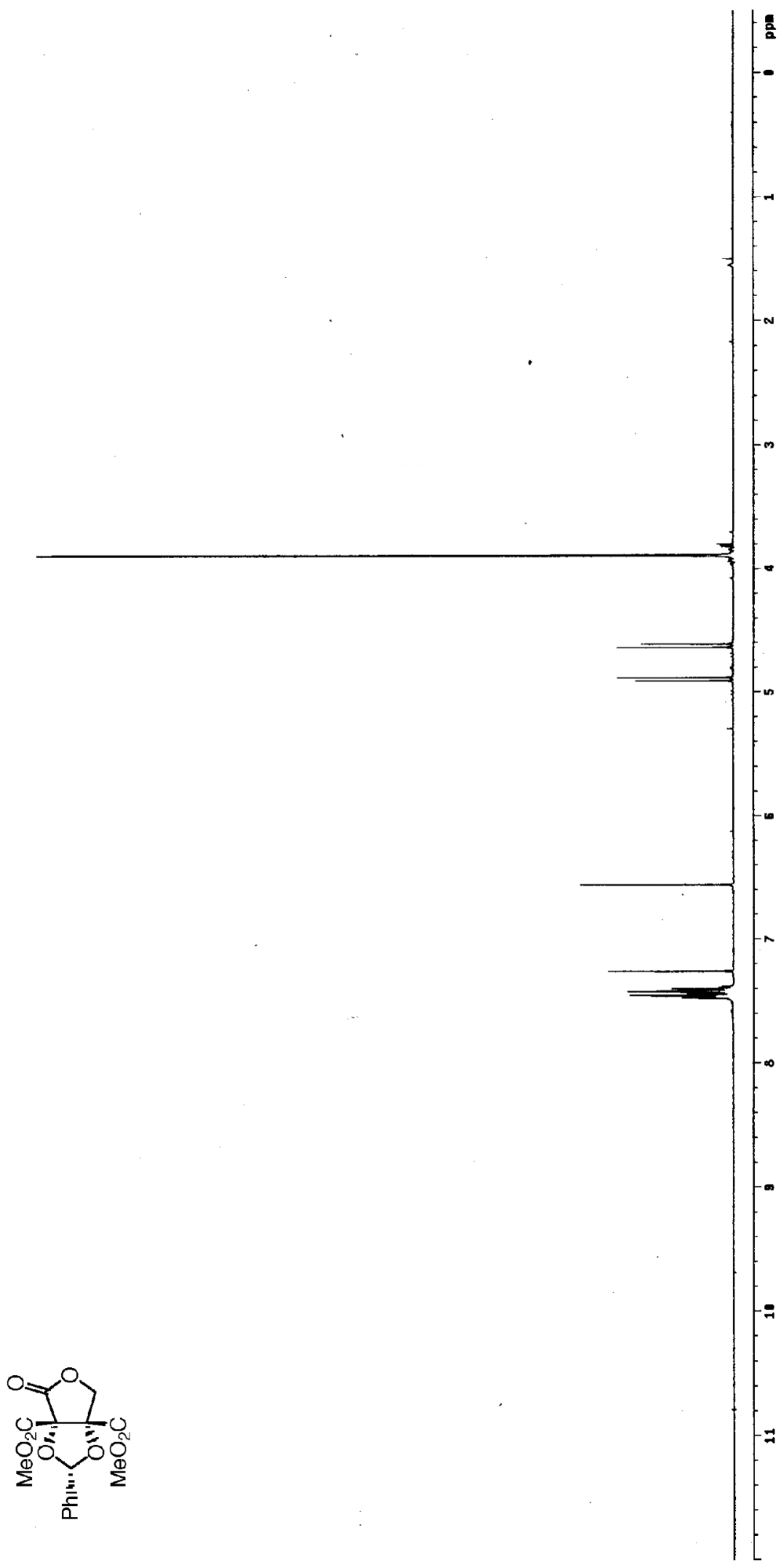



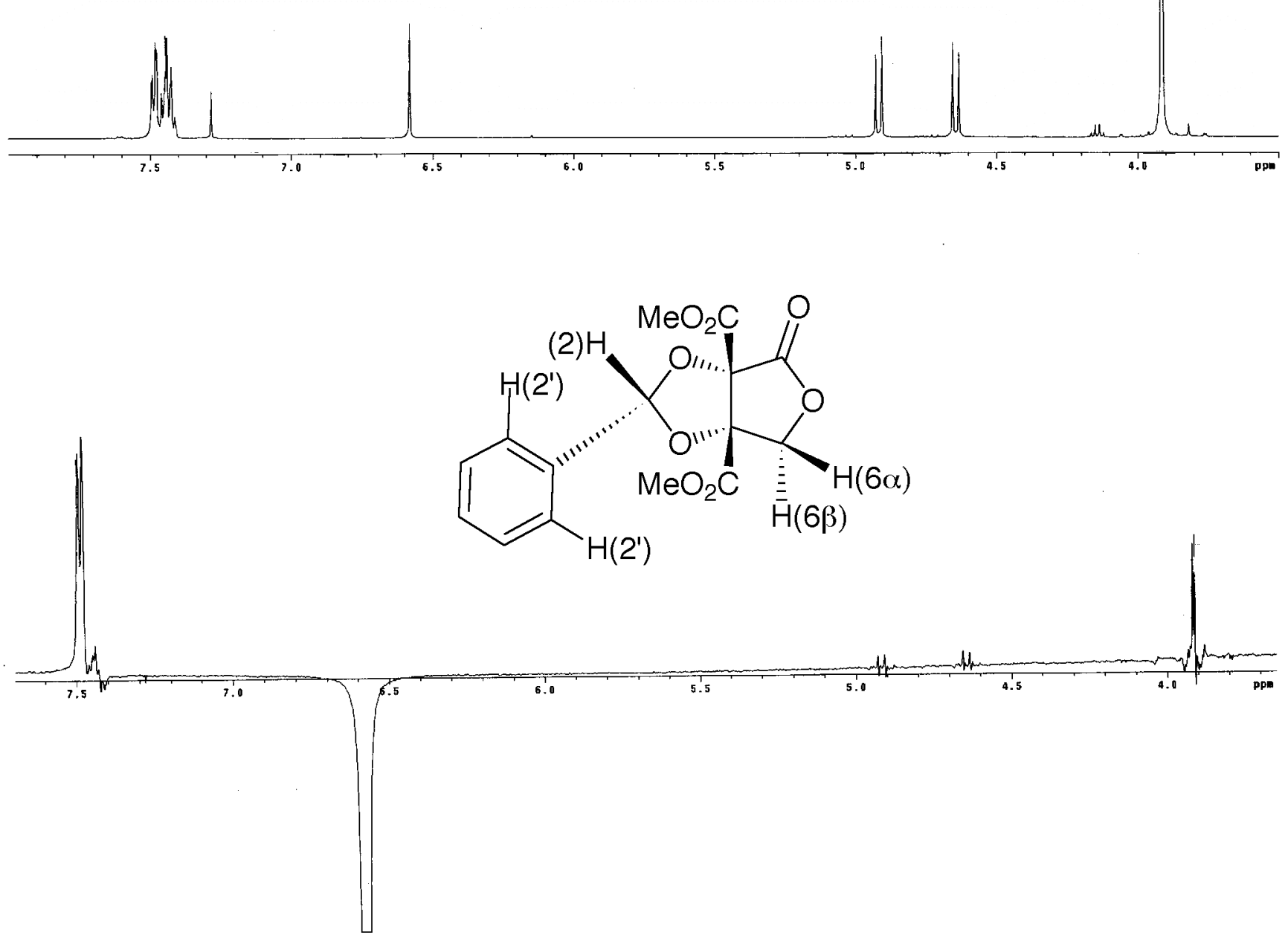

Figure 2: NOe Difference Spectra for 47.

A strong nOe is observed between $\mathrm{H} 2(\mathrm{~s}, 6.58 \mathrm{ppm})$ and $\mathrm{H} 2$ ' $(\mathrm{d}, 7.49 \mathrm{ppm})$ and a weak nOe is observed between $\mathrm{H} 2(6.58$ $\mathrm{ppm}$ ) and the methyl ester protons (3.910 and $3.915 \mathrm{ppm})$. The lack of a significant (compare Figure 1, page S14) interaction between $\mathrm{H} 2$ and $\mathrm{H} 6 \beta$ combined with the weak nOe between $\mathrm{H} 2$ and the methyl ester protons is indicative of the (2S)benzylidene stereoisomer. 
(2R,3aR,6aS)-(+)-4-tert-Butoxycarbonylmethyl-3a,6a-bis(methoxycarbonyl)-2phenyltetrahydrofuro[3,4- $d][1,3]$ dioxol-4-ol (48).

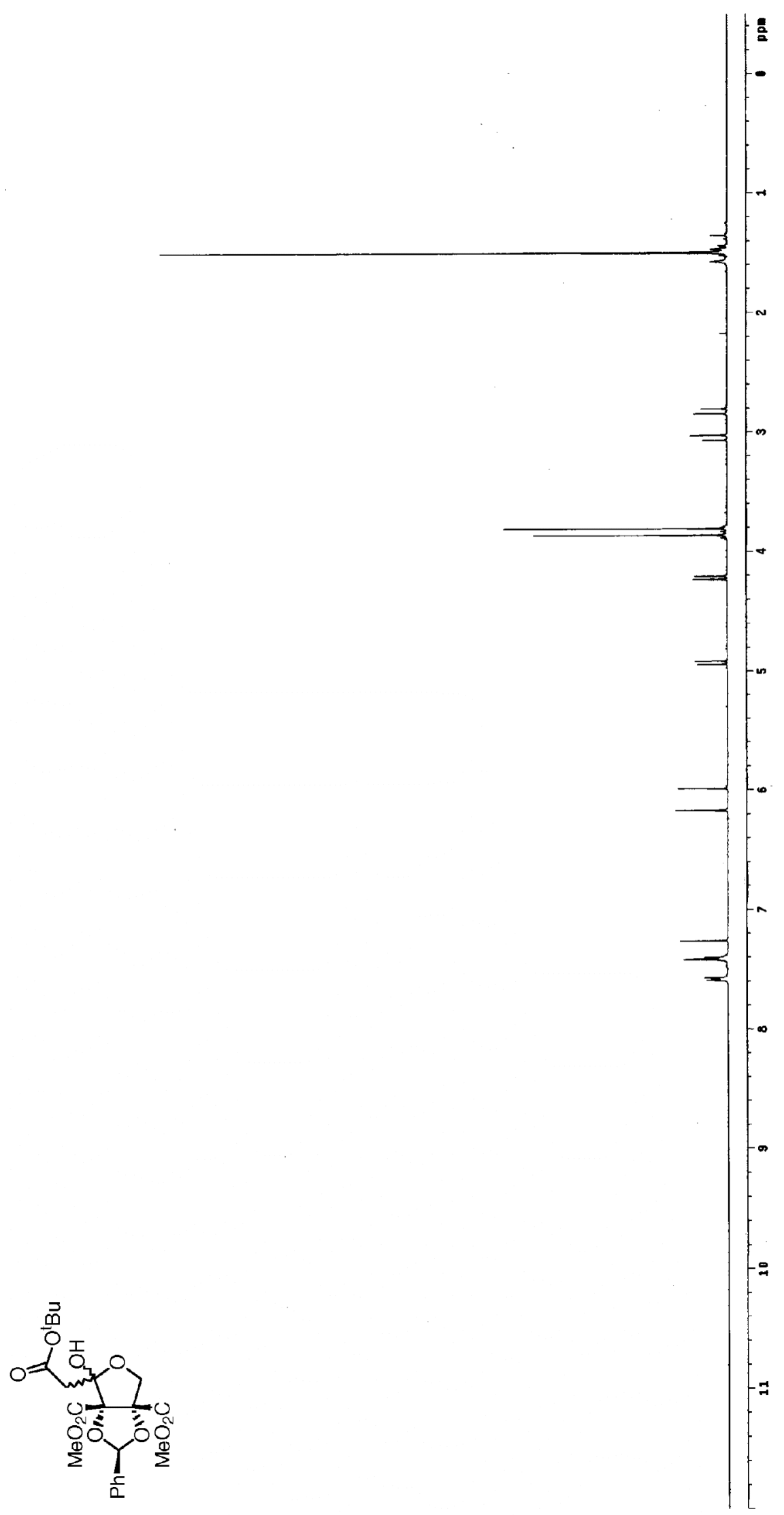


(2S,3aR,6aS)-(+)-4-tert-Butoxycarbonylmethyl-3a,6a-bis(methoxycarbonyl)-2phenyltetrahydrofuro[3,4- $d][1,3]$ dioxol-4-ol (49).

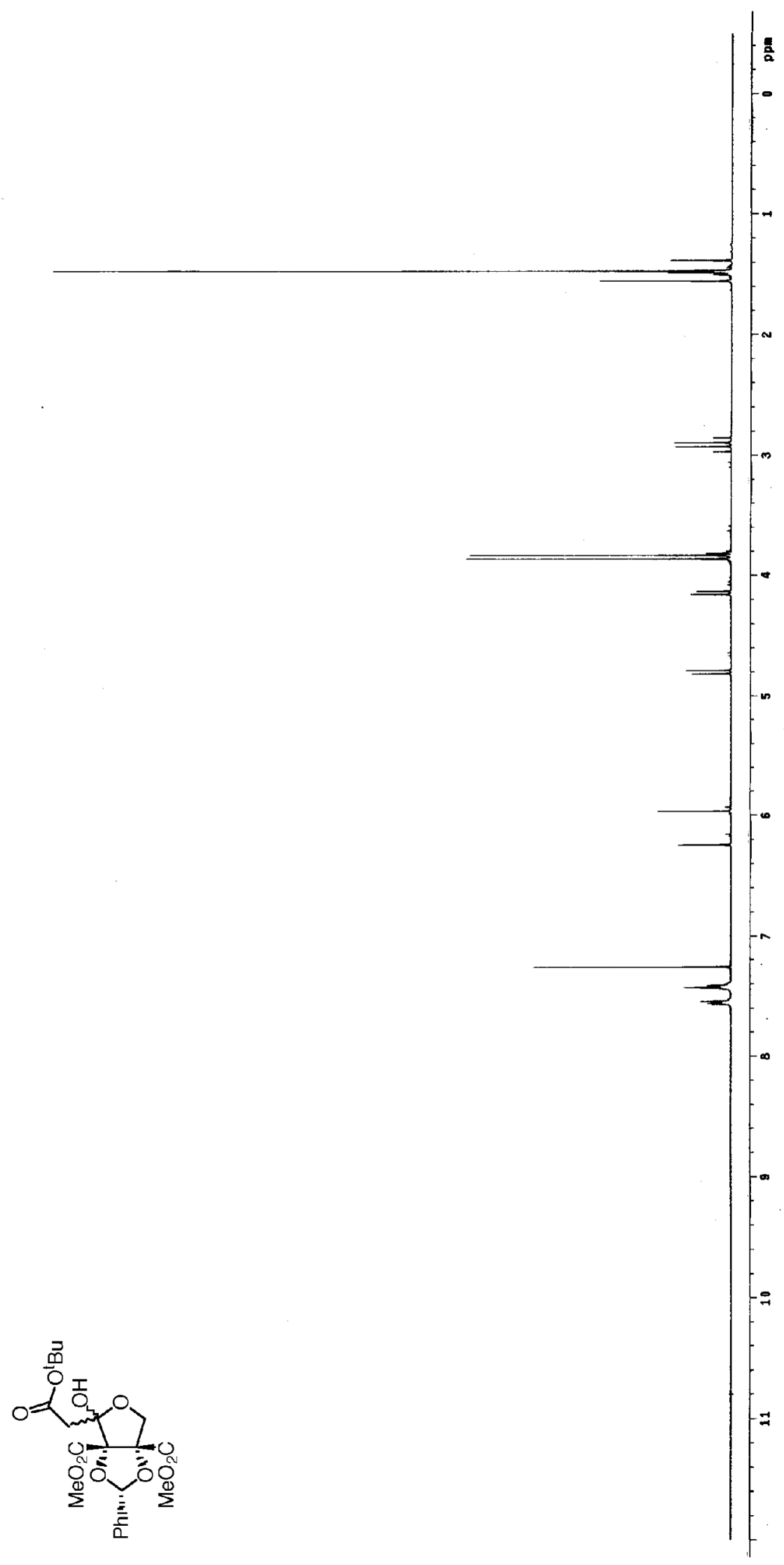


(3S,3aS-cis)-(+)-3-Hydroxy-6a-methoxy-3,3a-bis(methoxycarbonyl)tetrahydrofuro[2,3-b]furan-5-one (5).

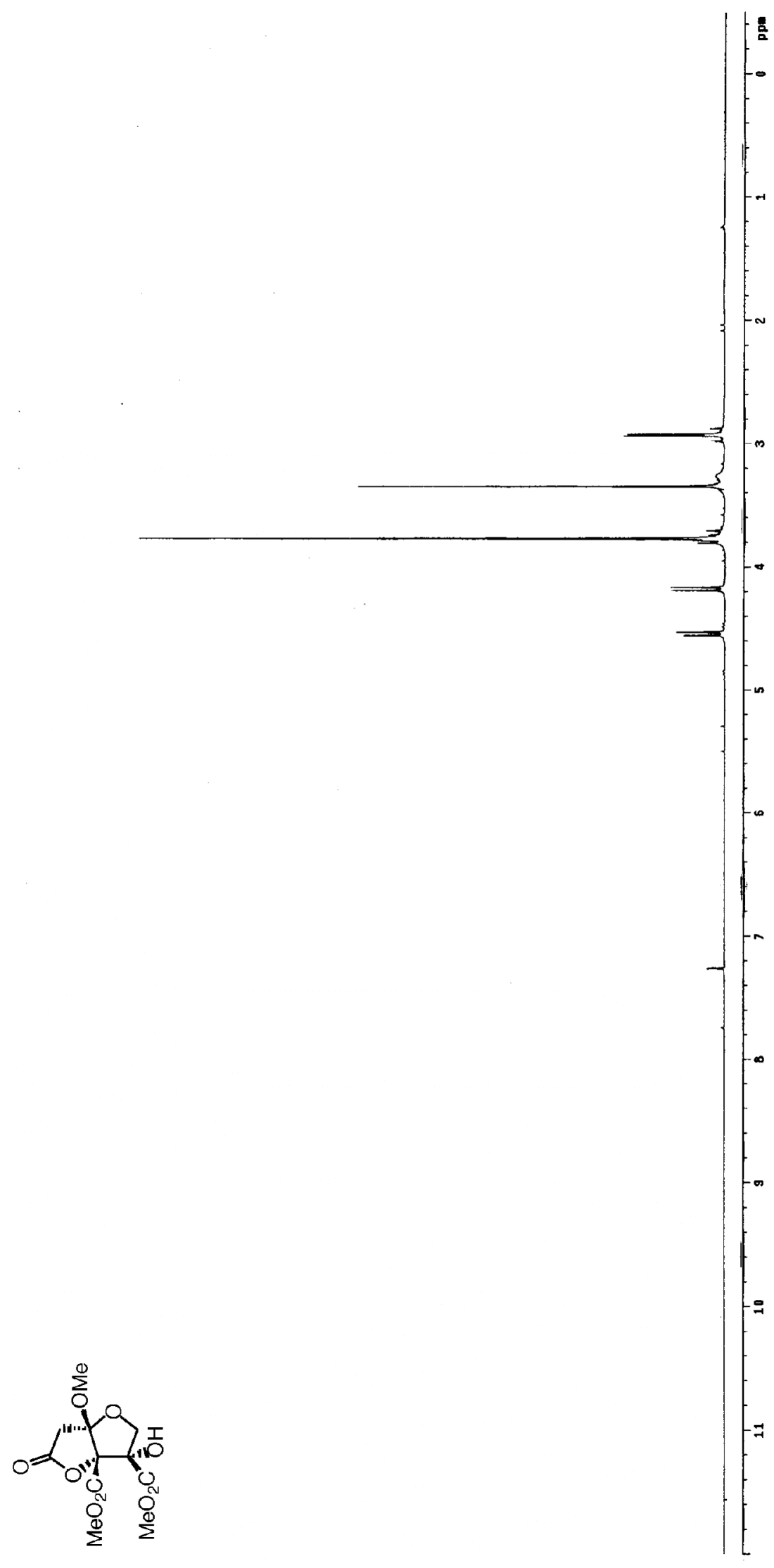




\section{$\underline{\text { Secondary Studies }}$}

\section{Experimental Section.}

(4S,5S)-4-Benzyloxymethyl-2,2-dimethyl-4,5-bis(methoxycarbonyl)[1,3]dioxolane (12).<smiles>COC[C@@H]1OC(C)(C)O[C@@H]1OC</smiles>

LDA, prepared by addition (over $5 \mathrm{~min}$ ) of a $2.50 \mathrm{M}$ solution of $n$-butyllithium in hexanes (4.40 $\mathrm{mL}, 11.0 \mathrm{mmol})$ to a solution of DIPA $(1.82 \mathrm{~mL}, 13.0 \mathrm{mmol})$ in THF $(50 \mathrm{~mL})$ at $-78{ }^{\circ} \mathrm{C}$, was added dropwise over $1 \mathrm{~h}$ via cannula to a solution of acetonide $10(2.18 \mathrm{~g}, 10.0 \mathrm{mmol})$ and $\mathrm{BOM}-\mathrm{Cl}(3.60 \mathrm{~g}$, $23.0 \mathrm{mmol})$ in a 1:5 (v/v) mixture of HMPA:THF $(60 \mathrm{~mL})$ at $-78{ }^{\circ} \mathrm{C}$. After addition was completed, the reaction was allowed to slowly warm to $-5{ }^{\circ} \mathrm{C}$ overnight. The reaction was quenched by addition of EtOAc $(150 \mathrm{~mL})$ followed by addition of water $(150 \mathrm{~mL})$. The organic layer was separated, washed with water $(3 \times 100 \mathrm{~mL})$ and brine $(3 \times 100 \mathrm{~mL})$, dried over $\mathrm{MgSO}_{4}$ and evaporated. Unreacted starting materials were removed via reduced-pressure $(0.1 \mathrm{~mm} \mathrm{Hg})$ distillation (oil bath temp $145{ }^{\circ} \mathrm{C}$ ). The crude residue was purified by flash chromatography using silica gel (Column, 5 x $16 \mathrm{~cm}$ ) with 4:1 hexanes:EtOAc as the eluent to yield a yellow oil $(2.03,60 \%) .{ }^{1} \mathrm{H}$ NMR $\left(400 \mathrm{MHz}, \mathrm{CDCl}_{3}, \delta\right): 1.43$ (s, $3 \mathrm{H}), 1.60(\mathrm{~s}, 3 \mathrm{H}), 3.63(\mathrm{~s}, 3 \mathrm{H}), 3.72-3.73($ apparent ABq, 2H), 3.83 (s, 3H), 4.49-4.51 (apparent ABq, $2 \mathrm{H}), 5.13(\mathrm{~s}, 1 \mathrm{H}), 7.24-7.31(\mathrm{~m}, 5 \mathrm{H}) .{ }^{13} \mathrm{C} \mathrm{NMR}$ (100 MHz, $\left.\mathrm{CDCl}_{3}, \delta\right): 25.7$ (1C), 27.2 (1C), 52.0 (1C), 52.8 (1C), 69.8 (1C), 73.3 (1C), 77.2 (1C), 84.9 (1C), 112.2 (1C), 127.0 (2C), 127.2 (1C), 127.9 (2C), 137.1 (1C), 168.2 (1C), 170.1 (1C). HRMS m/z: $[\mathrm{M}+\mathrm{Na}]^{+}$calcd for $\mathrm{C}_{17} \mathrm{H}_{22} \mathrm{O}_{7} \mathrm{Na}, 361.1263$; found, 361.1265.

(4S)-4-Benzyloxymethyl-2,2-dimethyl-4,5,5-tris(methoxycarbonyl)[1,3]dioxolane (14).

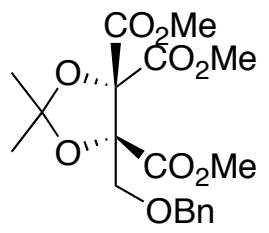

A solution of $12(1.74 \mathrm{~g}, 5.14 \mathrm{mmol})$ in THF $(15 \mathrm{~mL})$ at $-78{ }^{\circ} \mathrm{C}$ was added dropwise over 15 min to a solution of LDA, prepared by addition (over $5 \mathrm{~min}$ ) of a $2.50 \mathrm{M}$ solution of $n$-butyllithium in hexanes $(2.6 \mathrm{~mL}, 6.5 \mathrm{mmol})$ to a solution of DIPA $(1.0 \mathrm{~mL}, 7.1 \mathrm{mmol})$ in THF $(15 \mathrm{~mL})$ at $-78{ }^{\circ} \mathrm{C}$. After $30 \mathrm{~min}$, methyl chloroformate $(0.50 \mathrm{~mL}, 6.5 \mathrm{mmol})$ was added dropwise over 1 minute and the red solution turned a yellow-orange. After $45 \mathrm{~min}$, addition methyl chloroformate $(0.50 \mathrm{~mL}, 6.5 \mathrm{mmol})$ was added. After an additional $2 \mathrm{~h}$, a saturated solution of $\mathrm{NH}_{4} \mathrm{Cl}(25 \mathrm{~mL})$ was added and the resulting mixture was extracted with EtOAc $(3 \times 25 \mathrm{~mL})$. The organic extracts were combined, dried over $\mathrm{MgSO}_{4}$ and evaporated to yield an orange oil $(2.3 \mathrm{~g})$. The crude product was purified by flash chromatography using silica gel (Column, $5 \times 16 \mathrm{~cm}$ ) with 3:1 hexanes:EtOAc as the eluent to yield a yellow oil $(0.915 \mathrm{~g}, 45 \%)$. ${ }^{1} \mathrm{H}$ NMR $\left(400 \mathrm{MHz}, \mathrm{CDCl}_{3}, \delta\right): 1.58(\mathrm{~s}, 3 \mathrm{H}), 1.59(\mathrm{~s}, 3 \mathrm{H}), 3.72(\mathrm{~s}, 3 \mathrm{H}), 3,78$ $(\mathrm{s}, 3 \mathrm{H}), 3,79(\mathrm{~s}, 3 \mathrm{H}), 3.86(\mathrm{~d}, J=10.4 \mathrm{~Hz}, 1 \mathrm{H}), 4.00(\mathrm{~d}, J=10.4 \mathrm{~Hz}, 1 \mathrm{H}), 4.54-4.55(\mathrm{~m}, 2 \mathrm{H}), 7.25-7.31$ $(\mathrm{m}, 5 \mathrm{H}) .{ }^{13} \mathrm{C}$ NMR $\left(100 \mathrm{MHz}, \mathrm{CDCl}_{3}, \delta\right): 27.2$ (1C), 28.1 (1C), 52.7 (1C), 53.1 (1C), 53.4 (1C), 71.3 (1C), 73.5 (1C), 87.4 (1C), 88.8 (1C), 114.6 (1C), 127.2 (2C), 127.4 (1C), 128.1 (2C), 137.2 (1C), 166.0 (1C), 166.8 (1C), 169.0 (1C). HRMS m/z: [M+Na $]^{+}$calcd for $\mathrm{C}_{19} \mathrm{H}_{24} \mathrm{O}_{9} \mathrm{Na}, 419.1318$; found, 419.1314. 


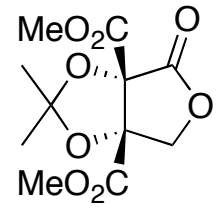

Triester $14(0.906 \mathrm{~g}, 2.29 \mathrm{mmol})$ and $10 \% \mathrm{Pd} / \mathrm{C}(0.140 \mathrm{~g})$ were stirred as a suspension in anhydrous $\mathrm{MeOH}(30 \mathrm{~mL})$ under a balloon of $\mathrm{H}_{2}$ for $16 \mathrm{~h}$. The crude reaction mixture was then vacuum filtered through a plug of celite and the filtrate was evaporated to yield an orange oil that solidifies upon cooling $(0.557 \mathrm{~g}, 89 \%)$. X-ray quality crystals were grown from a mixture of chloroform and pentane. ${ }^{1} \mathrm{H}$ NMR $\left(400 \mathrm{MHz} \mathrm{CDCl}_{3}, \delta\right): 1.52(\mathrm{~s}, 3 \mathrm{H}), 1.61(\mathrm{~s}, 3 \mathrm{H}), 3.83(\mathrm{~s}, 3 \mathrm{H}), 3.86$ $(\mathrm{s}, 3 \mathrm{H}), 4.54(\mathrm{~d}, J=10.8 \mathrm{~Hz}, 1 \mathrm{H}), 4.76(\mathrm{~d}, J=10.8 \mathrm{~Hz}, 1 \mathrm{H}) .{ }^{13} \mathrm{C} \mathrm{NMR}\left(100 \mathrm{MHz}, \mathrm{CDCl}_{3}, \delta\right): 27.0(1 \mathrm{C})$, 27.6 (1C), 53.5 (1C), 53.7 (1C), 71.5 (1C), 86.9 (1C), 88.8 (1C), 117.7 (1C), 163.7 (1C), 166.8 (1C), 170.0 (1C). HRMS m/z: [M+Na] $]^{+}$calcd for $\mathrm{C}_{11} \mathrm{H}_{14} \mathrm{O}_{8} \mathrm{Na}$, 297.0586; found, 297.0589.

(3aR-cis)-4-tert-Butoxycarbonylmethyl-2,2-dimethyl-3a,6a-bis(methoxycarbonyl)tetrahydrofuro[3,4d] [1,3]dioxol-4-ol (17).

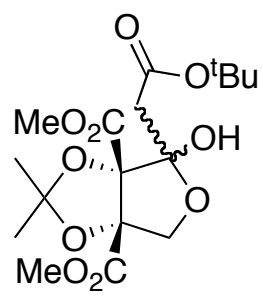

$t$-Butyl acetate $(0.11 \mathrm{~mL}, 0.82 \mathrm{mmol})$ was added dropwise over $5 \mathrm{~min}$ to a solution of LDA, prepared by addition (over $5 \mathrm{~min}$ ) of a $2.5 \mathrm{M}$ solution of $n$-butyllithium in hexanes $(0.26 \mathrm{~mL}, 0.66$ mmol $)$ to a solution of DIPA $(0.10 \mathrm{~mL}, 0.72 \mathrm{mmol})$ in THF $(2 \mathrm{~mL})$ at $-78{ }^{\circ} \mathrm{C}$. After one hour the lithium $t$-butyl acetate solution was cannulated into a $-78{ }^{\circ} \mathrm{C}$ solution of lactone $16(0.15 \mathrm{~g}, 0.55 \mathrm{mmol})$ in THF $(2 \mathrm{~mL})$. After three hours, the reaction was quenched by addition of saturated $\mathrm{NH}_{4} \mathrm{Cl}(5 \mathrm{~mL})$ and extracted with EtOAc $(3 \times 10 \mathrm{~mL})$. The organic extracts were combined, dried over $\mathrm{MgSO}_{4}$, and evaporated to yield an orange oil $(0.188 \mathrm{~g})$. The crude product was partially purified (see text and footnote 17) by flash chromatography using silica gel (Column, 3 x $16 \mathrm{~cm}$ ) with 3:1 hexanes:EtOAc as the eluent to yield an orange liquid $(0.100 \mathrm{~g})$. Compound 17 was never obtained entirely pure.

(4S,5S)-2,2-Dicyclopropyl-4,5-bis(methoxycarbonyl)[1,3]dioxolane. ${ }^{15}$

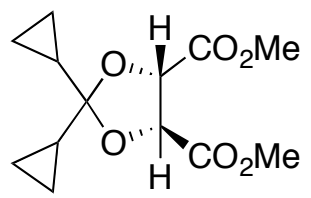

Dimethyl- D-tartrate $(\mathbf{8}, 25.0 \mathrm{~g}, 109 \mathrm{mmol})$ and CSA $(2.53 \mathrm{~g}, 10.9 \mathrm{mmol})$ were dissolved in DCM (315 mL). A crude 1:1 mixture of dicyclopropyl ketone and dicyclopropyl ketone dimethyl acetal (25.6 g) was added and the reaction was stirred for $5 \mathrm{~h}$ at ambient temperature. After the reaction was complete, the volatiles were removed by rotary evaporation and the resulting brown residue ( $35 \mathrm{~g})$ was fractionally distilled at $1 \mathrm{~mm} \mathrm{Hg}$. Of the two fractions collected, the more volatile $\left(40-50{ }^{\circ} \mathrm{C}\right)$ corresponded to a mixture of recovered dicyclopropyl ketone and dicyclopropyl ketone dimethyl acetal

\footnotetext{
${ }^{15}$ (a) Protection of tartrate with $\mathbf{2 7}$ was accomplished in $46 \%$ yield according to the general method described in Leighton, J. L. Ph.D. Thesis, Harvard University, Cambridge, MA, 1994, p123. (b) Hydrolytic cleavage of bis(cyclopropyl)formylidene ketals has been found to be over 100 times faster than acetonide cleavage: See Leighton, J. L. Ph.D. Thesis, Harvard University, Cambridge, MA, 1994, p 88.
} 
$(9 \mathrm{~g})$, while the less volatile fraction $\left(125-128^{\circ} \mathrm{C}\right)$ was found to be the desired ketal contaminated with a small amount (ca. 10\%) of unreacted starting material (13.5 g, ca. 46\%). An analytical sample was obtained by purifying a portion $(0.800 \mathrm{~g})$ of the distillate by flash chromatography using silica gel (Column, $3 \times 16 \mathrm{~cm}$ ) with 2:1 hexanes:EtOAc as the eluent to obtain a clear liquid $(0.605 \mathrm{~g}) .{ }^{1} \mathrm{H}$ NMR $\left(400 \mathrm{MHz}, \mathrm{CDCl}_{3}, \delta\right): 0.32-0.46(\mathrm{~m}, 6 \mathrm{H}), 0.54-0.62(\mathrm{~m}, 2 \mathrm{H}), 1.14-1.22(\mathrm{~m}, 2 \mathrm{H}), 3.76(\mathrm{~s}, 6 \mathrm{H}), 4.72(\mathrm{~s}$, 2H). ${ }^{13} \mathrm{C} \mathrm{NMR}\left(100 \mathrm{MHz}, \mathrm{CDCl}_{3}, \delta\right): 1.41$ (2C), 1.43 (2C), 17.9 (2C), 52.6 (2C), 77.3 (2C), 114.9 (1C), 169.4 (2C). HRMS m/z: [M+Na] $]^{+}$calcd for $\mathrm{C}_{13} \mathrm{H}_{18} \mathrm{O}_{6} \mathrm{Na}, 293.1001$; found, 293.1003.

(4S,5S)-2-Cyclopropyl-4,5-bis(methoxycarbonyl)-2-methyl[1,3]dioxolane. ${ }^{16}$

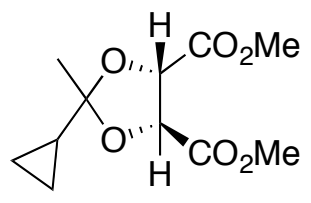

Dimethyl-D-tartrate $(8,2.50 \mathrm{~g}, 14.0 \mathrm{mmol})$ and p-toluenesulfonic acid $(0.040 \mathrm{~g}, 0.14 \mathrm{mmol})$ were dissolved in a mixture of anhydrous benzene $(10 \mathrm{~mL})$ and cyclopropyl methyl ketone $(1.32 \mathrm{~mL}, 14.0$ $\mathrm{mmol}$ ). The reaction was heated to reflux and stirred overnight with excess water being removed from the reaction via a Dean-Stark apparatus. The reaction was allowed to cool to ambient temperature and the solvent was removed by rotary evaporation. The crude product was purified by flash chromatography using silica gel with 2:1 hexanes:EtOAc as the eluent to yield the desired ketal (1.19 g, $35 \%) .{ }^{1} \mathrm{H}$ NMR $\left(400 \mathrm{MHz}, \mathrm{CDCl}_{3}, \delta\right): 0.30-0.60(\mathrm{~m}, 4 \mathrm{H}), 1.05-1.25(\mathrm{~m}, 1 \mathrm{H}), 1.51(\mathrm{~s}, 3 \mathrm{H}), 3.82(\mathrm{~s}$, $3 \mathrm{H}), 3.83(\mathrm{~s}, 3 \mathrm{H}), 4.81(\mathrm{~s}, 2 \mathrm{H}) .{ }^{13} \mathrm{C}$ NMR $\left(100 \mathrm{MHz}, \mathrm{CDCl}_{3}, \delta\right): 1.8(1 \mathrm{C}), 1.9(1 \mathrm{C}), 18.8(1 \mathrm{C}), 24.9$ (1C), 52.7 (1C), 71.9 (1C), 77.0 (1C), 77.2 (1C), 114.5 (1C), 169.5 (1C), 169.8 (1C). HRMS m/z: $[\mathrm{M}+\mathrm{Na}]^{+}$calcd for $\mathrm{C}_{11} \mathrm{H}_{16} \mathrm{O}_{6} \mathrm{Na}, 267.0845$; found, 267.0844 .

(3a'R-cis)-3a',6a'-Bis(methoxycarbonyl)spiro(cyclopentane-1,2'-dihydrofuro[3,4-d][1,3]dioxol)4'(3a'H)-one (34).

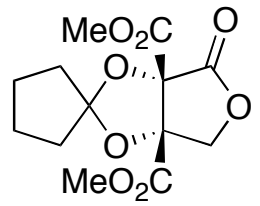

Triester $32(2.25 \mathrm{~g}, 5.30 \mathrm{mmol})$ and $10 \% \mathrm{Pd} / \mathrm{C}(225 \mathrm{mg})$ were stirred as a suspension in anhydrous $\mathrm{MeOH}(60 \mathrm{~mL})$ under a balloon of $\mathrm{H}_{2}$ for 3 hours. Additional $10 \% \mathrm{Pd} / \mathrm{C}(225 \mathrm{mg})$ was added and the reaction was stirred under $\mathrm{H}_{2}$ overnight. The crude reaction mixture was then filtered through a plug of celite and the filtrate was evaporated to yield an orange oil $(1.59 \mathrm{~g}, 100 \%)$. ${ }^{1} \mathrm{H}$ NMR (400 MHz, $\left.\mathrm{CDCl}_{3}, \delta\right): 2.6-2.8(\mathrm{~m}, 4 \mathrm{H}), 2.8-2.0(\mathrm{~m}, 2 \mathrm{H}), 2.0-2.1(\mathrm{~m}, 2 \mathrm{H}), 3.81(\mathrm{~s}, 3 \mathrm{H}), 3.85(\mathrm{~s}, 3 \mathrm{H})$, $4.54(\mathrm{~d}, J=10.8 \mathrm{~Hz}, 1 \mathrm{H}), 4.80(\mathrm{~d}, J=10.8 \mathrm{~Hz}, 1 \mathrm{H}) .{ }^{13} \mathrm{C} \mathrm{NMR}\left(100 \mathrm{MHz}, \mathrm{CDCl}_{3}, \delta\right): 23.3(1 \mathrm{C}), 23.4$ (1C), 37.1 (1C), 37.6 (1C), 53.4 (1C), 53.6 (1C), 71.3 (1C), 86.8 (1C), 88.2 (1C), 126.7 (1C), 163.4 (1C), 166.5 (1C), 169.7 (1C). HRMS m/z: [M+Na] ${ }^{+}$calcd for $\mathrm{C}_{13} \mathrm{H}_{16} \mathrm{O}_{8} \mathrm{Na}, 323.0743$; found, 323.0739.

\footnotetext{
${ }^{16}$ Protection of tartrate with $\mathbf{2 9}$ was accomplished in $35 \%$ yield according to the general method described in Sylvestre, J.; Marc, J.; Song-Yu, T.; Patrick, G. Bull. Soc. Chim. Fr. 1964, 12, 3207-3217.
} 
(3aR-cis)-4-tert-Butoxycarbonylmethyl-3a,6a-bis(methoxycarbonyl)spiro(cyclopentane-1,2'tetrahydrofuro[3,4-d][1,3]dioxol)-4'-ol (37).<smiles>COC1(OC)CCCC1</smiles>

$t$-Butyl acetate $(1.0 \mathrm{~mL}, 7.4 \mathrm{mmol})$ was dissolved in $\mathrm{Et}_{2} \mathrm{O}(10 \mathrm{~mL})$ and cooled to $-78{ }^{\circ} \mathrm{C}$. A 0.49 $\mathrm{M}^{17}$ solution of LDA (13.8 mL. $\left.6.76 \mathrm{mmol}\right)$, prepared by addition (over $10 \mathrm{~min}$ ) of a $2.5 \mathrm{M}$ solution of $n$-butyllithium in hexanes $(2.80 \mathrm{~mL}, 7.0 \mathrm{mmol})$ to a solution of DIPA $(1.0 \mathrm{~mL}, 7.1 \mathrm{mmol})$ in $\mathrm{Et}_{2} \mathrm{O}(10$ $\mathrm{mL})$ at $-78{ }^{\circ} \mathrm{C}$, was added dropwise over $10 \mathrm{~min}$. In a second reaction flask, 34 (1.60 g, $\left.5.33 \mathrm{mmol}\right)$ was stirred as a solution in $\mathrm{Et}_{2} \mathrm{O}(41 \mathrm{~mL})$. $\mathrm{TiCl}_{4}(5.5 \mathrm{~mL}, 1.0 \mathrm{M}$ in DCM, $5.5 \mathrm{mmol})$ was added in one portion, the yellow solution was cooled to $-78{ }^{\circ} \mathrm{C}$ and part of the lithium $t$-butyl acetate stock solution $(22 \mathrm{~mL}, 0.3 \mathrm{M}, 6.6 \mathrm{mmol}$ ) was added dropwise over $20 \mathrm{~min}$. After $1 \mathrm{~h}$, the reaction was quenched by pouring the cold reaction mixture into a saturated solution of $\mathrm{NH}_{4} \mathrm{Cl}(60 \mathrm{~mL})$ and the aqueous layer was extracted with $\mathrm{Et}_{2} \mathrm{O}(2 \times 40 \mathrm{~mL})$. The organic extracts were combined, dried over $\mathrm{MgSO}_{4}$ and evaporated to yield a yellow oil $(2.55 \mathrm{~g})$. The crude product was purified by flash chromatography using silica gel (Column, $5 \times 16 \mathrm{~cm}$ ) with 3:1 hexanes:EtOAc as the eluent to yield a mixture of regioisomers $(1.26 \mathrm{~g}, 57 \%)$ resulting from attack at the lactone carbonyl and attack at one of the two ester functionalities. Chromatography using silica gel (Column, $5 \times 16 \mathrm{~cm}$ ) with $1 \%$ EtOAc in DCM cleanly separates this mixture yielding 37 as a clear oil $(0.761 \mathrm{~g}, 34 \%)$. ${ }^{1} \mathrm{H}$ NMR $\left(400 \mathrm{MHz}, \mathrm{CDCl}_{3}, \delta\right): 1.49$ $(\mathrm{s}, 9 \mathrm{H}), 1.66-1.71(\mathrm{~m}, 4 \mathrm{H}), 1.99-2.05(\mathrm{~m}, 2 \mathrm{H}), 2.10-2.11(\mathrm{~m}, 2 \mathrm{H}), 2.84(\mathrm{~d}, J=16 \mathrm{~Hz}, 1 \mathrm{H}), 2.95(\mathrm{~d}, J=$ $16 \mathrm{~Hz}, 1 \mathrm{H}), 3.79(\mathrm{~s}, 3 \mathrm{H}), 3.83(\mathrm{~s}, 3 \mathrm{H}), 3.97(\mathrm{~d}, J=10 \mathrm{~Hz}, 1 \mathrm{H}), 4.77(\mathrm{~d}, J=10 \mathrm{~Hz}, 1 \mathrm{H}), 5.86(\mathrm{~s}, 1 \mathrm{H}) .{ }^{13} \mathrm{C}$ NMR (100 MHz, $\mathrm{CDCl}_{3}, \delta$ ): 14.3 (1C), 22.7 (1C), 23.8 (1C), 28.1 (3C), 36.8 (1C), 36.9 (1C), 38.3 (1C), 60.4 (1C), 74.0 (1C), 82.6 (1C), 92.6 (1C), 96.8 (1C), 105.1 (1C), 125.9 (1C), 167.6 (1C), 169.1 (1C), 171.3 (1C). HRMS m/z: [M+Na] $]^{+}$calcd for $\mathrm{C}_{19} \mathrm{H}_{28} \mathrm{O}_{10} \mathrm{Na}, 439.1580$; found, 439.1597 .

(3aR-cis)-Dihydro-3a,6a-bis(methoxycarbonyl)furo[3,4- $d][1,3]$ dioxol-4(3aH)-one (43).

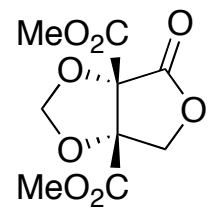

Lactone $38(0.500 \mathrm{~g}, 2.14 \mathrm{mmol})$ was dissolved in a mixture of DCM (10 $\mathrm{mL})$ and dimethoxymethane $(10.0 \mathrm{~mL}, 113 \mathrm{mmol})$. Phosphorous pentoxide $(5.0 \mathrm{~g}, 35 \mathrm{mmol})$ was added and the reaction mixture was heated at reflux overnight. After cooling to $0{ }^{\circ} \mathrm{C}$, the reaction was quenched by addition of water $(20 \mathrm{~mL})$ over $15 \mathrm{~min}$ and the aqueous layer was extracted with DCM $(3 \times 25 \mathrm{~mL})$. The organic extracts were combined, washed with saturated $\mathrm{NaHCO}_{3}(1 \mathrm{x} 10 \mathrm{~mL})$, dried over $\mathrm{MgSO}_{4}$ and evaporated to yield a dark oil $(1.06 \mathrm{~g})$. The crude product was purified by flash chromatography using silica gel (Column, $3 \times 16 \mathrm{~cm}$ ) with 2:1 hexanes:EtOAc as the eluent to yield $43(0.520 \mathrm{~g}, 99 \%)$. ${ }^{1} \mathrm{H}$ NMR (400 MHz, $\left.\mathrm{CDCl}_{3}, \delta\right): 3.83(\mathrm{~s}, 3 \mathrm{H}), 3.87(\mathrm{~s}, 3 \mathrm{H}), 4.59(\mathrm{~d}, J=10.8 \mathrm{~Hz}, 1 \mathrm{H}), 4.88(\mathrm{~d}, J=10.8$ $\mathrm{Hz}, 1 \mathrm{H}), 5.41(\mathrm{~s}, 1 \mathrm{H}), 5.51(\mathrm{~s}, 1 \mathrm{H}) .{ }^{13} \mathrm{C} \mathrm{NMR}\left(100 \mathrm{MHz}, \mathrm{CDCl}_{3}, \delta\right): 53.6$ (1C), 53.8 (1C), 70.5 (1C), 86.6 (1C), 88.5 (1C), 99.7 (1C), 163.6 (1C), 165.8 (1C), 168.2 (1C). HRMS m/z: [M+Na] calcd for $\mathrm{C}_{9} \mathrm{H}_{10} \mathrm{O}_{8} \mathrm{Na}, 269.0273$; found, 269.0277.

\footnotetext{
${ }^{17}$ Titrated using the method described in Duhamel, L.; Plaquevent, J. J. Organomet. Chem. 1993, 448, 1-3.
} 
(3aR-cis)-4-tert-Butoxycarbonylmethyl-3a,6a-bis(methoxycarbonyl)tetrahydrofuro[3,4- $d][1,3]$ dioxol-4ol (45).<smiles>COC[C@]12CO[C@](O)(CC(=O)OC)[C@](COC)(OCO1)[C@H]2OC</smiles>

$t$-Butyl acetate $(0.700 \mathrm{~mL}, 5.19 \mathrm{mmol})$ was dissolved in $\mathrm{Et}_{2} \mathrm{O}(40 \mathrm{~mL})$ and cooled to $-78{ }^{\circ} \mathrm{C}$. A $0.48 \mathrm{M}^{18}$ solution of LDA (10.4 mL. $4.99 \mathrm{mmol}$ ), prepared by addition (over $10 \mathrm{~min}$ ) of a $2.5 \mathrm{M}$ solution of $n$-butyllithium in hexanes $(7.70 \mathrm{~mL}, 19.2 \mathrm{mmol})$ to a solution of DIPA $(2.70 \mathrm{~mL}, 19.3$ $\mathrm{mmol})$ in $\mathrm{Et}_{2} \mathrm{O}(30 \mathrm{~mL})$ at $-78{ }^{\circ} \mathrm{C}$, was added dropwise over $10 \mathrm{~min}$. In a second reaction flask, 43 $(0.246 \mathrm{~g}, 1.0 \mathrm{mmol})$ was stirred as a suspension in $\mathrm{Et}_{2} \mathrm{O}(10 \mathrm{~mL})$ that dissolves upon addition of $\mathrm{TiCl}_{4}$ $\left(1.0 \mathrm{~mL}, 1.0 \mathrm{M}\right.$ in DCM, $1.0 \mathrm{mmol}$ ). This solution was cooled to $-78{ }^{\circ} \mathrm{C}$ and part of the lithium $t$-butyl acetate stock solution $(15 \mathrm{~mL}, 0.1 \mathrm{M}, 1.5 \mathrm{mmol})$ was added dropwise over $20 \mathrm{~min}$. After one hour, the reaction was quenched by pouring the cold reaction mixture into a saturated solution of $\mathrm{NH}_{4} \mathrm{Cl}(30 \mathrm{~mL})$ and the aqueous layer was extracted with $\mathrm{Et}_{2} \mathrm{O}(2 \times 20 \mathrm{~mL})$. The organic extracts were combined, dried over $\mathrm{MgSO}_{4}$ and evaporated to yield a yellow oil $(0.382 \mathrm{~g})$. The crude product was purified by chromatography using silica gel (Column, $3 \times 16 \mathrm{~cm}$ ) with 3:1 hexanes:EtOAc as the eluent to yield a mixture of regioisomers $(0.135 \mathrm{~g}, 40 \%)$ resulting from attack either at the lactone carbonyl or at one of the two ester functionalities. Chromatography using silica gel with $1 \%$ EtOAc in DCM cleanly separates this mixture yielding 45 as a clear oil $(0.060 \mathrm{~g}, 15 \%)$. ${ }^{1} \mathrm{H}$ NMR $\left(400 \mathrm{MHz}, \mathrm{CDCl}_{3}, \delta\right): 1.45(\mathrm{~s}, 9 \mathrm{H})$, $2.81(\mathrm{~d}, J=16 \mathrm{~Hz}, 1 \mathrm{H}), 2.91(\mathrm{~d}, J=16 \mathrm{~Hz}, 1 \mathrm{H}), 3.78(\mathrm{~s}, 3 \mathrm{H}), 3.80(\mathrm{~s}, 3 \mathrm{H}), 4.01(\mathrm{~d}, J=10 \mathrm{~Hz}, 1 \mathrm{H}), 4.73$ $(\mathrm{d}, J=10 \mathrm{~Hz}, 1 \mathrm{H}), 5.23(\mathrm{~s}, 1 \mathrm{H}), 5.28(\mathrm{~s}, 1 \mathrm{H}), 5.89(\mathrm{~s}, 1 \mathrm{H}) .{ }^{13} \mathrm{C} \mathrm{NMR}\left(125 \mathrm{MHz}, \mathrm{CDCl}_{3}, \delta\right): 28.0(3 \mathrm{C})$, 38.5 (1C), 52.9 (1C), 53.0 (1C), 71.9 (1C), 82.7 (1C), 91.9 (1C), 95.9 (1C), 98.6 (1C), 105.0 (1C), 167.2 (1C), $168.5(1 \mathrm{C}), 171.3$ (1C). HRMS m/z: [M+Na $]^{+}$calcd for $\mathrm{C}_{15} \mathrm{H}_{22} \mathrm{O}_{10} \mathrm{Na}, 385.1111$; found, 385.1107.

(3R-cis)-Dihydro-3,4-bis(acetoxy)-3,4-bis(methoxycarbonyl)furan-2(3H)-one.

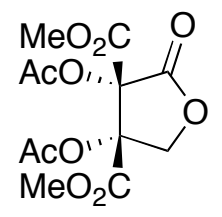

Lactone 38 (0.200 g, $0.850 \mathrm{mmol})$ was dissolved in neat acetyl chloride $(2.5 \mathrm{~mL}, 35 \mathrm{mmol})$ and the resulting solution was stirred at reflux overnight. After cooling to ambient temperature, the mixture was concentrated under vacuum $(0.1 \mathrm{~mm} \mathrm{Hg})$ and the residual oil was purified by chromatography using silica gel (Column, $3 \times 16 \mathrm{~cm}$ ) with 1:1 hexanes:EtOAc as the eluent to yield the desired bisprotected derivative $(0.247 \mathrm{~g}, 91 \%)$. ${ }^{1} \mathrm{H}$ NMR $\left(400 \mathrm{MHz} \mathrm{CDCl}_{3}, \delta\right): 2.20(\mathrm{~s}, 3 \mathrm{H}), 2.27(\mathrm{~s}, 3 \mathrm{H}), 3.80(\mathrm{~s}, 3 \mathrm{H}), 3.84$ $(\mathrm{s}, 3 \mathrm{H}), 4.65(\mathrm{~d}, J=11.6 \mathrm{~Hz}, 1 \mathrm{H}), 5.06(\mathrm{~d}, J=11.6 \mathrm{~Hz}, 1 \mathrm{H}) .{ }^{13} \mathrm{C} \mathrm{NMR}\left(100 \mathrm{MHz}, \mathrm{CDCl}_{3}, \delta\right): 20.5(1 \mathrm{C})$, 20.8 (1C), 53.7 (1C), 54.1 (1C), 70.3 (1C), 81.3 (1C), 82.4 (1C), 162.9 (1C), 164.6 (1C), 165.5 (1C), 168.0 (1C), 169.1 (1C). HRMS m/z: $[\mathrm{M}+\mathrm{Na}]^{+}$calcd for $\mathrm{C}_{12} \mathrm{H}_{14} \mathrm{O}_{10} \mathrm{Na}, 341.0485$; found, 341.0480 .

\footnotetext{
${ }^{18}$ Titrated using the method described in Duhamel, L.; Plaquevent, J. J. Organomet. Chem. 1993, 448, 1-3.
} 


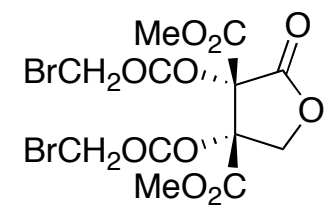

To a solution of lactone $38(0.300 \mathrm{~g}, 1.27 \mathrm{mmol})$ in DCM $(12.8 \mathrm{~mL})$ at $0{ }^{\circ} \mathrm{C}$ was added 2,6 lutidine $(0.64 \mathrm{~mL}, 5.28 \mathrm{mmol})$ followed by bromoacetyl bromide $(0.38 \mathrm{~mL}, 4.1 \mathrm{mmol})$. After stirring at $0{ }^{\circ} \mathrm{C}$ for $0.5 \mathrm{~h}$, the solution was allowed to warm to ambient temperature. After an additional $2 \mathrm{~h}$ the reaction mixture was neutralized (aqueous layer $\mathrm{pH}$ of 7 ) using $0.5 \mathrm{~N} \mathrm{HCl}$. The aqueous layer was extracted with EtOAc $(2 \times 10 \mathrm{~mL})$. The organic layers were combined, dried over sodium sulfate and evaporate. The crude product was purified by chromatography using silica gel (Column, $5 \mathrm{x} 16 \mathrm{~cm}$ ) with 3:2 hexanes:EtOAc as the eluent to yield both the monoprotected derivative $(0.508 \mathrm{mg}, 73 \%)$ and the desired bisprotected derivative $(0.160 \mathrm{mg}, 26 \%) .{ }^{1} \mathrm{H}$ NMR $\left(400 \mathrm{MHz}, \mathrm{CDCl}_{3}, \delta\right): 3.81(\mathrm{~s}, 3 \mathrm{H}), 3.86$ $(\mathrm{s}, 3 \mathrm{H}), 3.94(\mathrm{~s}, 2 \mathrm{H}), 4.02(\mathrm{~s}, 2 \mathrm{H}), 4.70(\mathrm{~d}, J=11.6 \mathrm{~Hz}, 1 \mathrm{H}), 5.08(\mathrm{~d}, J=11.6 \mathrm{~Hz}, 1 \mathrm{H}) .{ }^{13} \mathrm{C}$ NMR $(100$ $\left.\mathrm{MHz}, \mathrm{CDCl}_{3}, \delta\right): 24.4$ (1C), 24.5 (1C), 54.0 (1C), 54.5 (1C), 70.2 (1C), 81.8 (1C), 83.5 (1C), 161.9 (1C), 163.8 (1C), 164.2 (1C), 164.4 (1C), 165.4 (1C). HRMS m/z: [M+Na] calcd for $\mathrm{C}_{12} \mathrm{H}_{12} \mathrm{O}_{10} \mathrm{Br}_{2} \mathrm{Na}^{+}$, 496.8695; found, 496.8698 .

(3R-cis)-Dihydro-3,4-bis(methoxycarbonyl)-3,4-bis(trimethylsilanyloxy)furan-2(3H)-one.

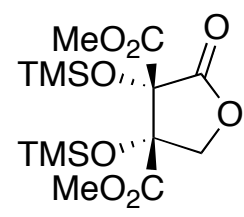

Lactone 38 (0.100 g, $0.425 \mathrm{mmol})$ was dissolved in THF (3 mL). Trimethylsilyl chloride $(0.163 \mathrm{~mL}, 1.70 \mathrm{mmol})$ and triethylamine $(0.140 \mathrm{~mL}, 1.70 \mathrm{mmol})$ were added and the reaction was stirred at ambient temperature overnight. After rotary evaporation of the volatiles, the residue was purified by flash chromatography using silica gel (Column, $3 \times 16 \mathrm{~cm}$ ) with 3:1 hexanes:EtOAc as the eluent to yield the desired bisprotected derivative as an oil $(0.153 \mathrm{~g}, 96 \%)$. ${ }^{1} \mathrm{H} \mathrm{NMR}\left(400 \mathrm{MHz}, \mathrm{CDCl}_{3}\right.$, ठ): $0.17(\mathrm{~s}, 9 \mathrm{H}), 0.23(\mathrm{~s}, 9 \mathrm{H}), 3.81(\mathrm{~s}, 3 \mathrm{H}), 3.84(\mathrm{~s}, 3 \mathrm{H}), 4.29(\mathrm{~d}, J=9.6 \mathrm{~Hz}, 1 \mathrm{H}), 4.81(\mathrm{~d}, J=9.6 \mathrm{~Hz}$, $1 \mathrm{H}) .{ }^{13} \mathrm{C} \mathrm{NMR}\left(100 \mathrm{MHz}, \mathrm{CDCl}_{3}, \delta\right): 1.8(3 \mathrm{C}), 2.0(3 \mathrm{C}), 52.8(1 \mathrm{C}), 53.2(1 \mathrm{C}), 74.8$ (1C), 83.8 (1C), 84.2 (1C), 167.6 (1C), 168.3 (1C), 170.9 (1C). HRMS m/z: [M+Na] calcd for $\mathrm{C}_{14} \mathrm{H}_{26} \mathrm{O}_{8} \mathrm{Si}_{2} \mathrm{Na}^{+}$, 401.1064; found, 401.1061. 


\section{${ }^{1}$ H-NMR spectra}

(4S,5S)-4-Benzyloxymethyl-2,2-dimethyl-4,5-bis(methoxycarbonyl)[1,3]dioxolane (12).

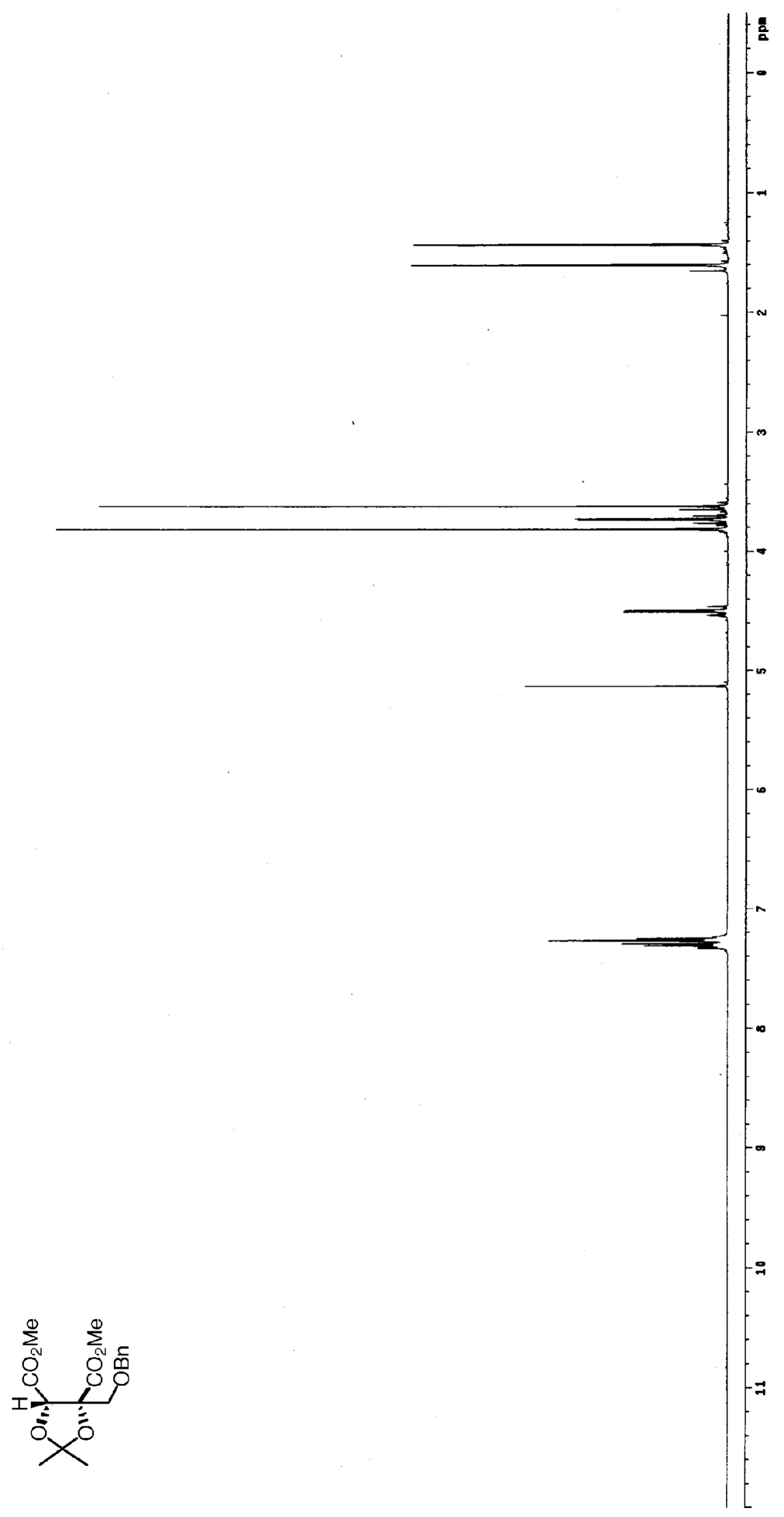




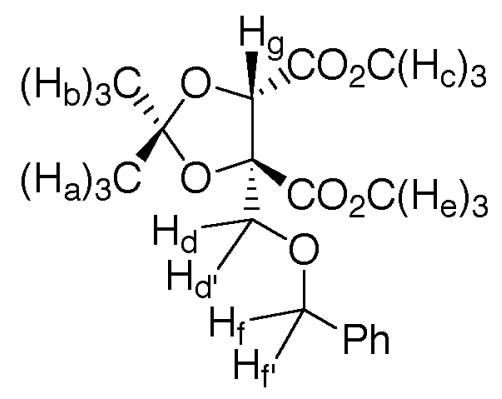

$\mathrm{H}_{\mathrm{a}}=1.43 \mathrm{ppm}$
$\mathrm{H}_{\mathrm{b}}=1.60 \mathrm{ppm}$
$\mathrm{H}_{\mathrm{c}}=3.63 \mathrm{ppm}$
$\mathrm{H}_{\mathrm{d} / \mathrm{d}^{\prime}}=3.73 \mathrm{ppm}$
$\mathrm{H}_{\mathrm{e}}=3.83 \mathrm{ppm}$
$\mathrm{H}_{\mathrm{fff}}=4.50 \mathrm{ppm}$
$\mathrm{H}_{\mathrm{g}}=5.13 \mathrm{ppm}$
$\mathrm{Ph}=7.24-7.30 \mathrm{ppm}$

$\mathrm{H}_{\mathrm{e}} \mid \mathrm{H}_{\mathrm{c}}$

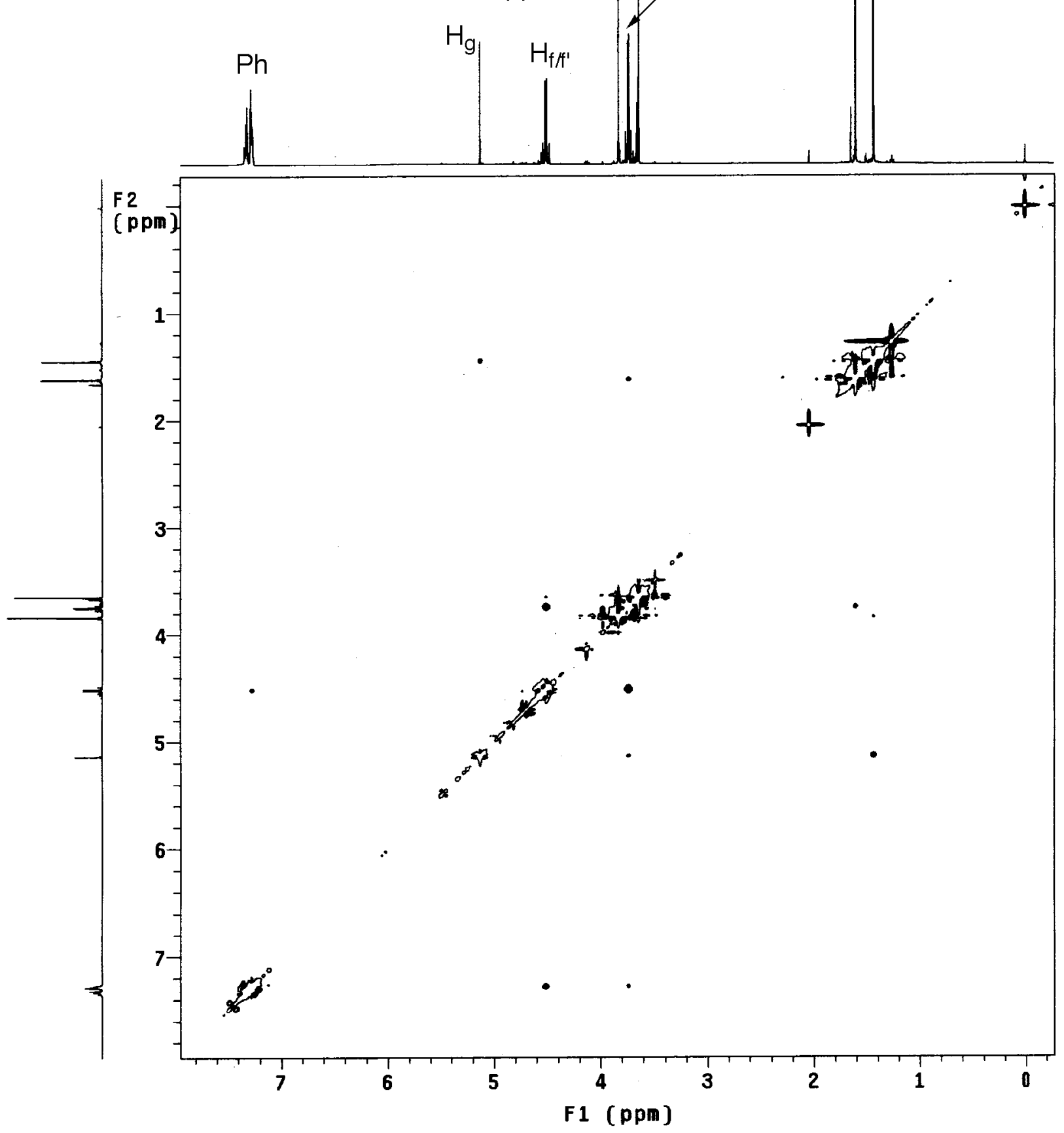

Figure 2: NOESY2D Spectra for 12.

Observed nOe's: $\mathrm{H}_{\mathrm{a}} \rightarrow \mathrm{H}_{\mathrm{e}}, \mathrm{H}_{\mathrm{g}} ; \mathrm{H}_{\mathrm{b}} \rightarrow \mathrm{H}_{\mathrm{d} / \mathrm{d}} ; \mathrm{H}_{\mathrm{d} / \mathrm{d}^{\prime}} \rightarrow \mathrm{H}_{\mathrm{b}}, \mathrm{H}_{\mathrm{fff}}, \mathrm{H}_{\mathrm{g}}, \mathrm{Ph} ; \mathrm{H}_{\mathrm{f} / \mathrm{f}^{\prime}} \rightarrow \mathrm{H}_{\mathrm{c}}, \mathrm{H}_{\mathrm{d} / \mathrm{d}^{\prime}}, \mathrm{Ph} ; \mathrm{H}_{\mathrm{g}} \rightarrow \mathrm{H}_{\mathrm{a}}$. The nOe observed between the $\mathrm{H}_{\mathrm{a}}$ acetonide methyl and $\mathrm{H}_{\mathrm{g}}$ combined with the nOe observed between the acetonide methyl on the other side of the ring $\left(\mathrm{H}_{\mathrm{b}}\right)$ and the $\mathrm{H}_{\mathrm{d} / \mathrm{d}}$, $\mathrm{AB}$ quartet is consistent with the expected trans diester diastereomer. It should be noted that modeling indicates that an nOe between $\mathrm{H}_{\mathrm{d} / \mathrm{d}}$ and $\mathrm{H}_{\mathrm{g}}$ could be observed in either the cis or trans isomer and is therefore not diagnostic. 
(4S)-4-Benzyloxymethyl-2,2-dimethyl-4,5,5-tris(methoxycarbonyl)[1,3]dioxolane (14).

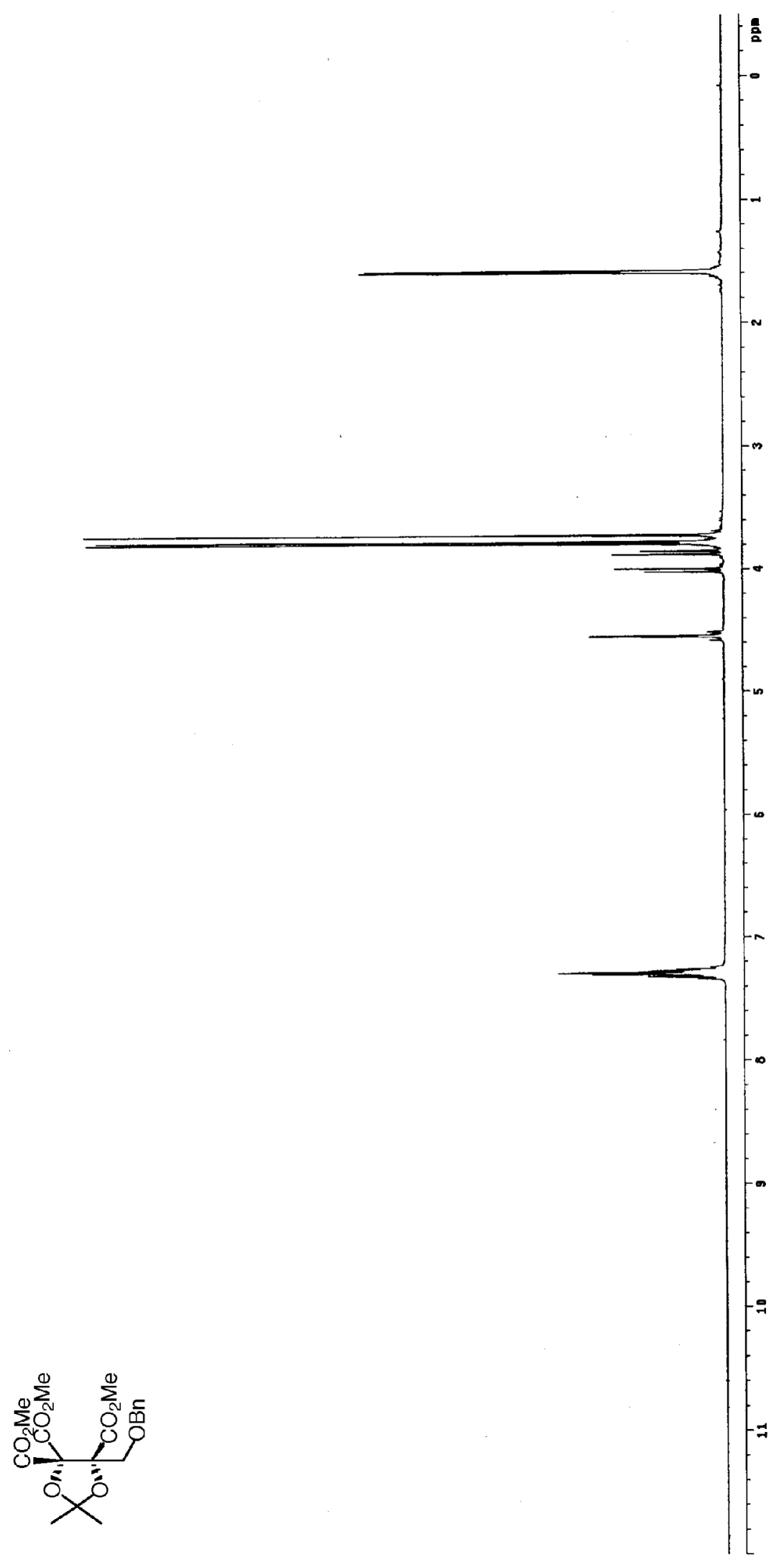


(3aR-cis)-Dihydro-2,2-dimethyl-3a,6a-bis(methoxycarbonyl)furo[3,4- $d][1,3]$ dioxol-4(3aH)-one (16).

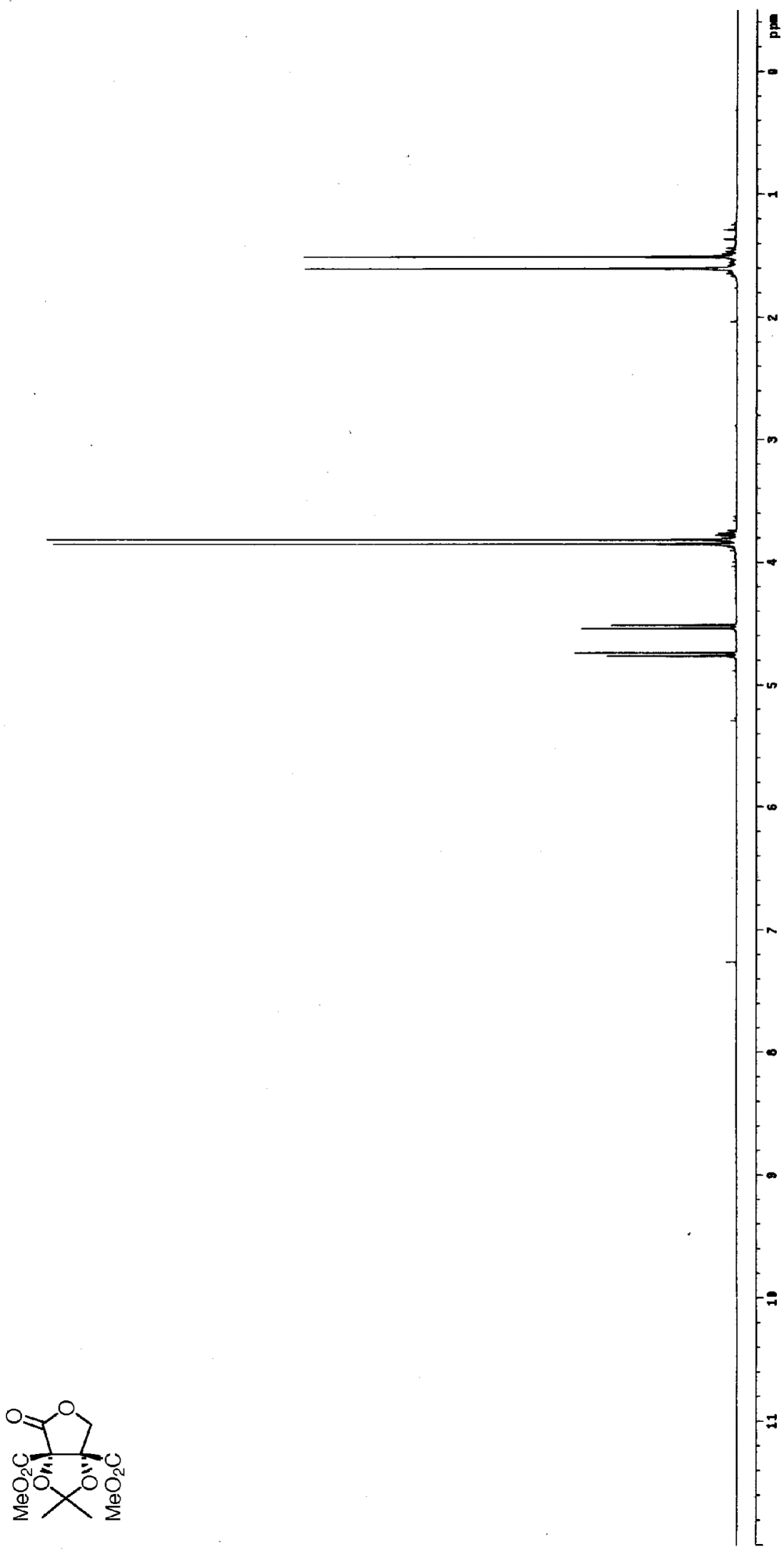


(3aR-cis)-4-tert-Butoxycarbonylmethyl-2,2-dimethyl-3a,6a-bis(methoxycarbonyl)tetrahydrofuro[3,4d][1,3]dioxol-4-ol (17).

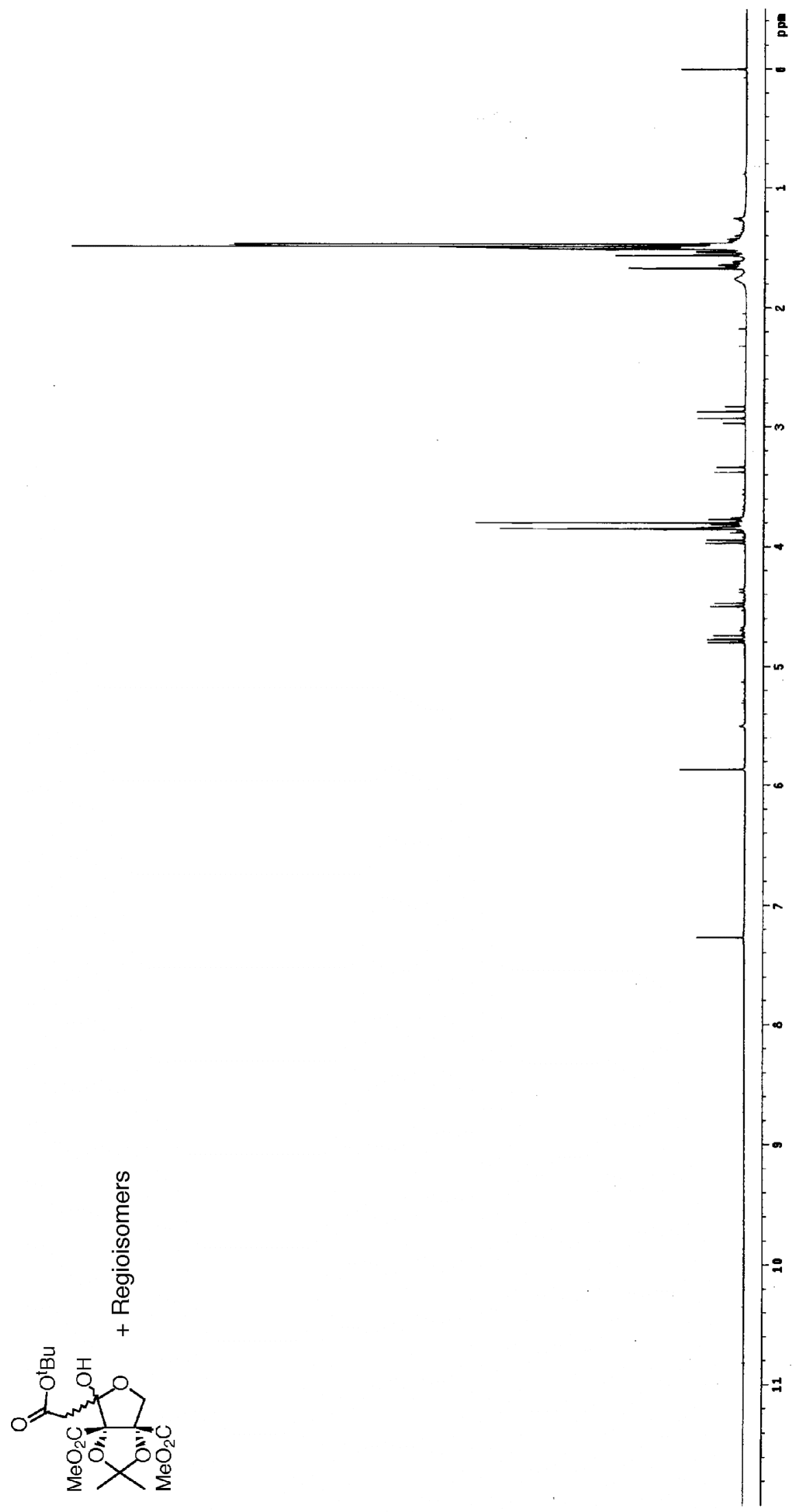


(4S,5S)-2,2-Dicyclopropyl-4,5-bis(methoxycarbonyl)[1,3]dioxolane.

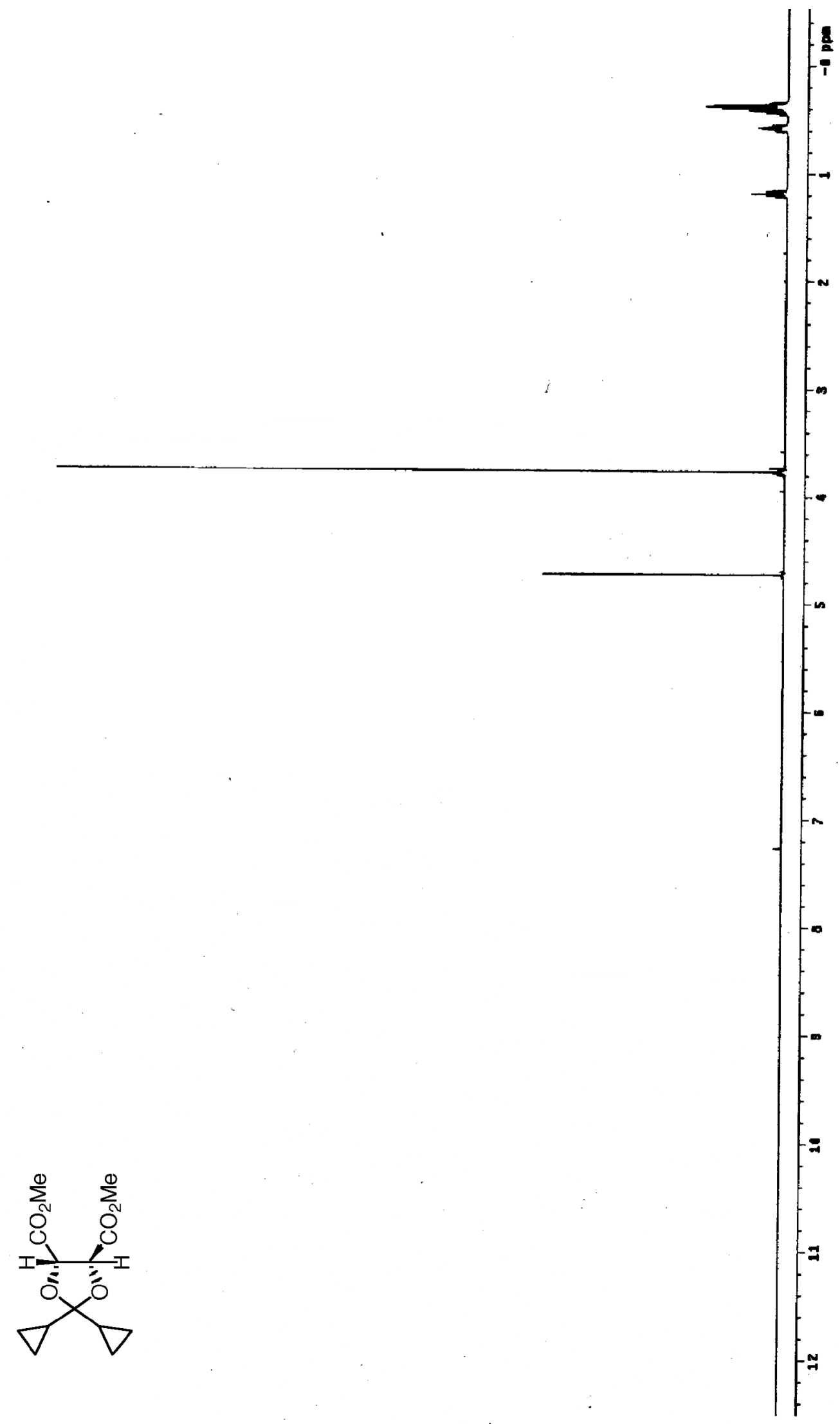


(4S,5S)-2-Cyclopropyl-4,5-bis(methoxycarbonyl)-2-methyl[1,3]dioxolane.

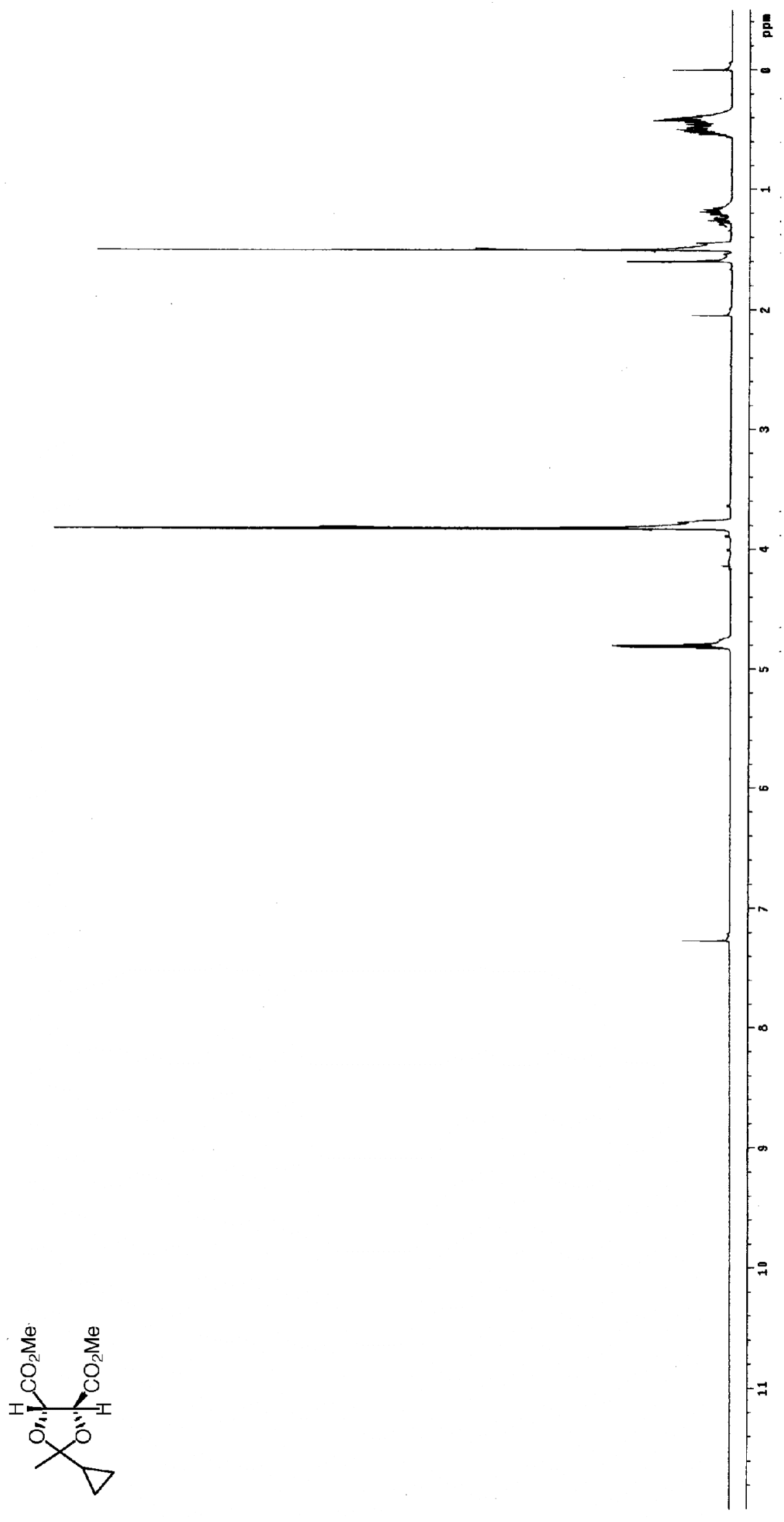


(3a'R-cis)-3a',6a'-Bis(methoxycarbonyl)spiro(cyclopentane-1,2'-dihydrofuro[3,4-d][1,3]dioxol)$\underline{4^{\prime}\left(3 a^{\prime} H\right)-o n e ~(34)}$.

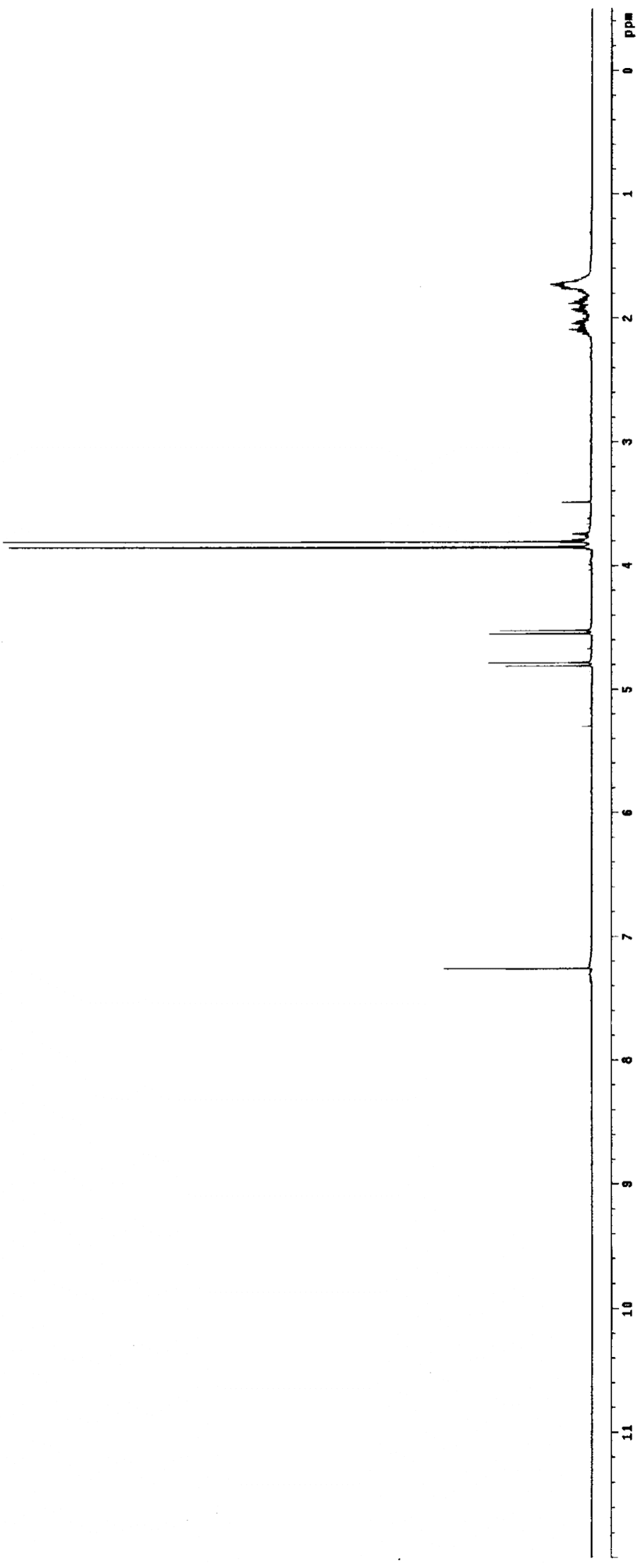


(3aR-cis)-4-tert-Butoxycarbonylmethyl-3a,6a-bis(methoxycarbonyl)spiro(cyclopentane-1,2'tetrahydrofuro[3,4-d][1,3]dioxol)-4'-ol (37).

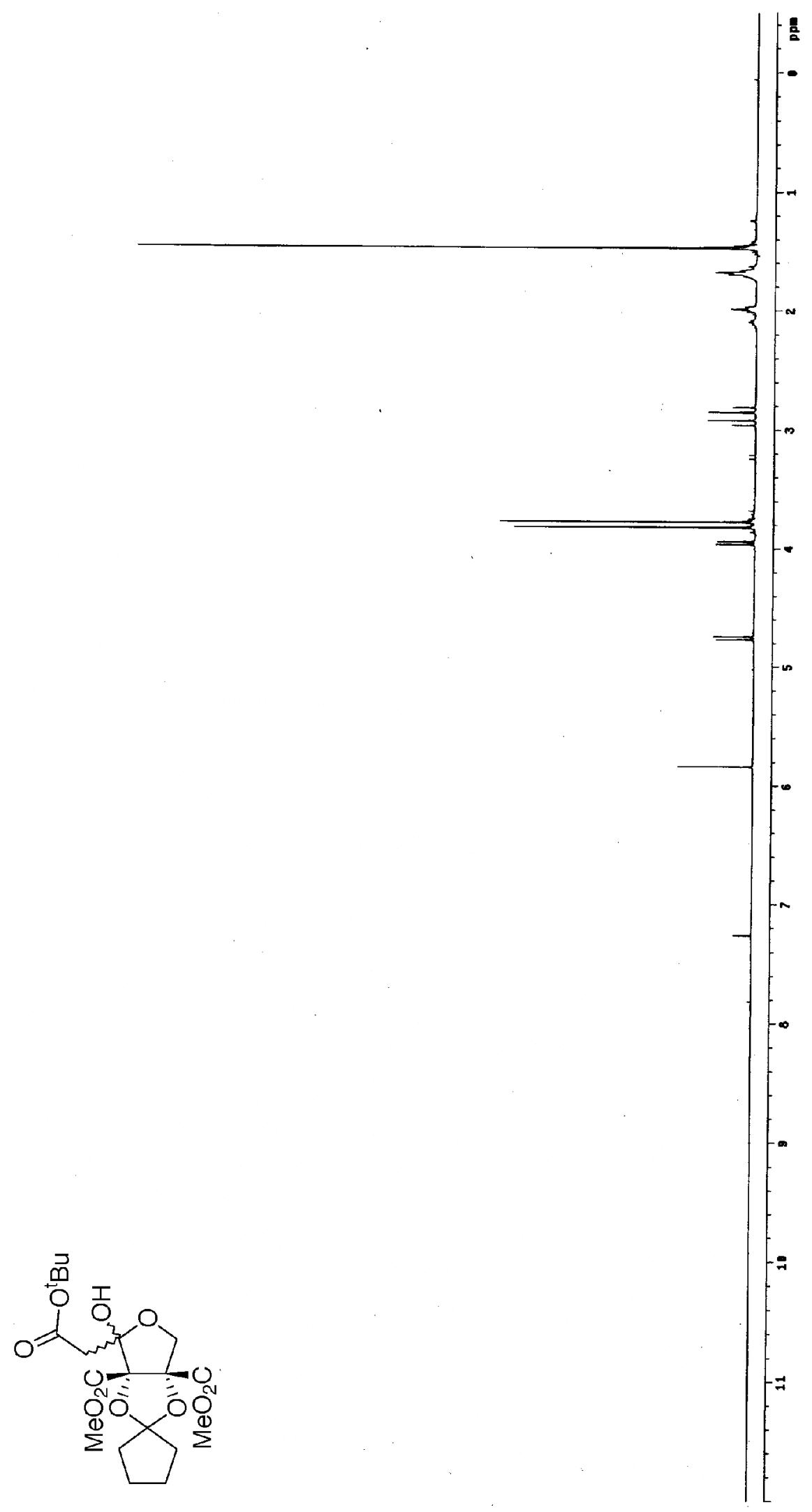


(3aR-cis)-Dihydro-3a,6a-bis(methoxycarbonyl)furo[3,4- $d][1,3]$ dioxol-4(3aH)-one (43).

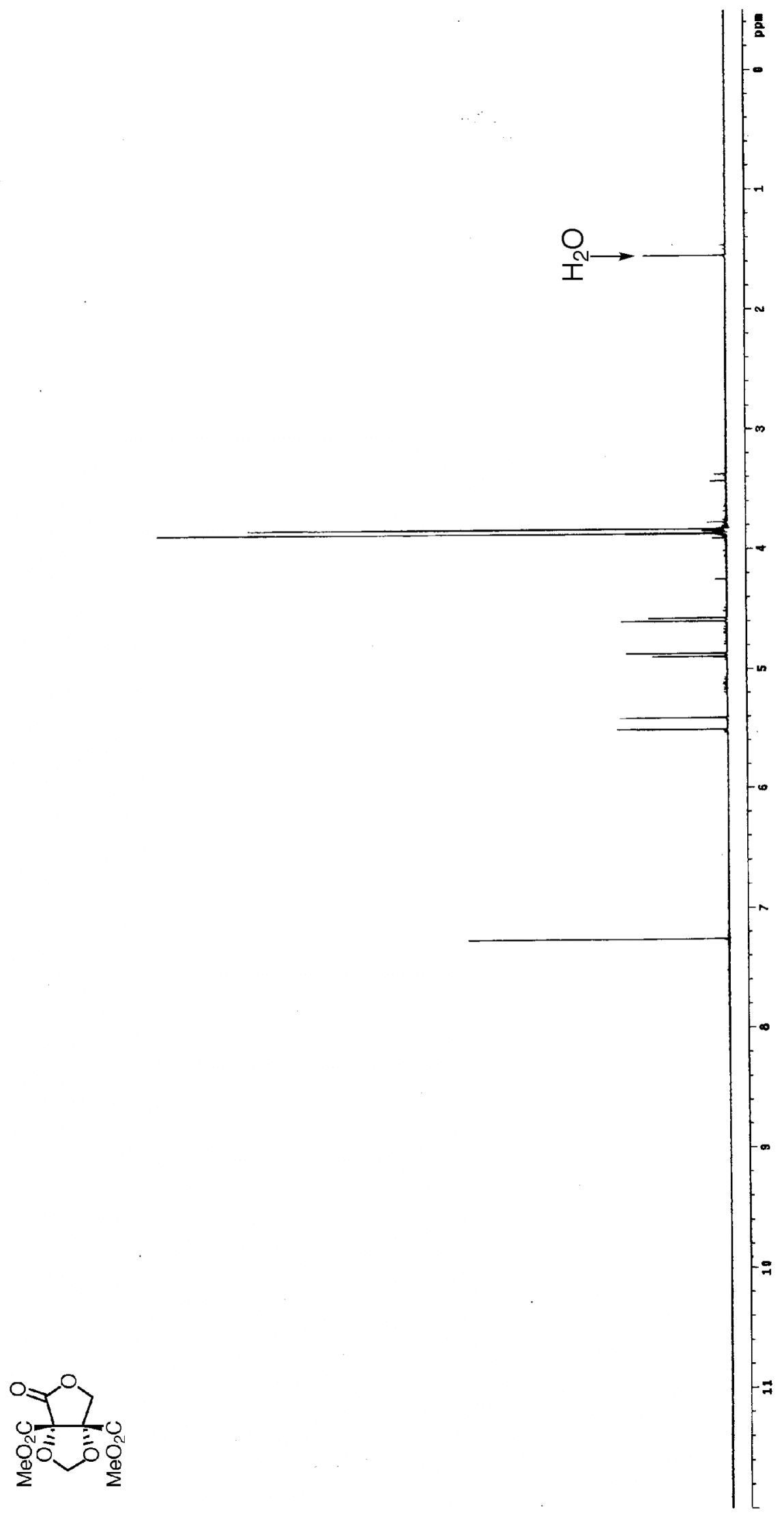


(3aR-cis)-4-tert-Butoxycarbonylmethyl-3a,6a-bis(methoxycarbonyl)tetrahydrofuro[3,4- $d][1,3]$ dioxol-4ol (45).

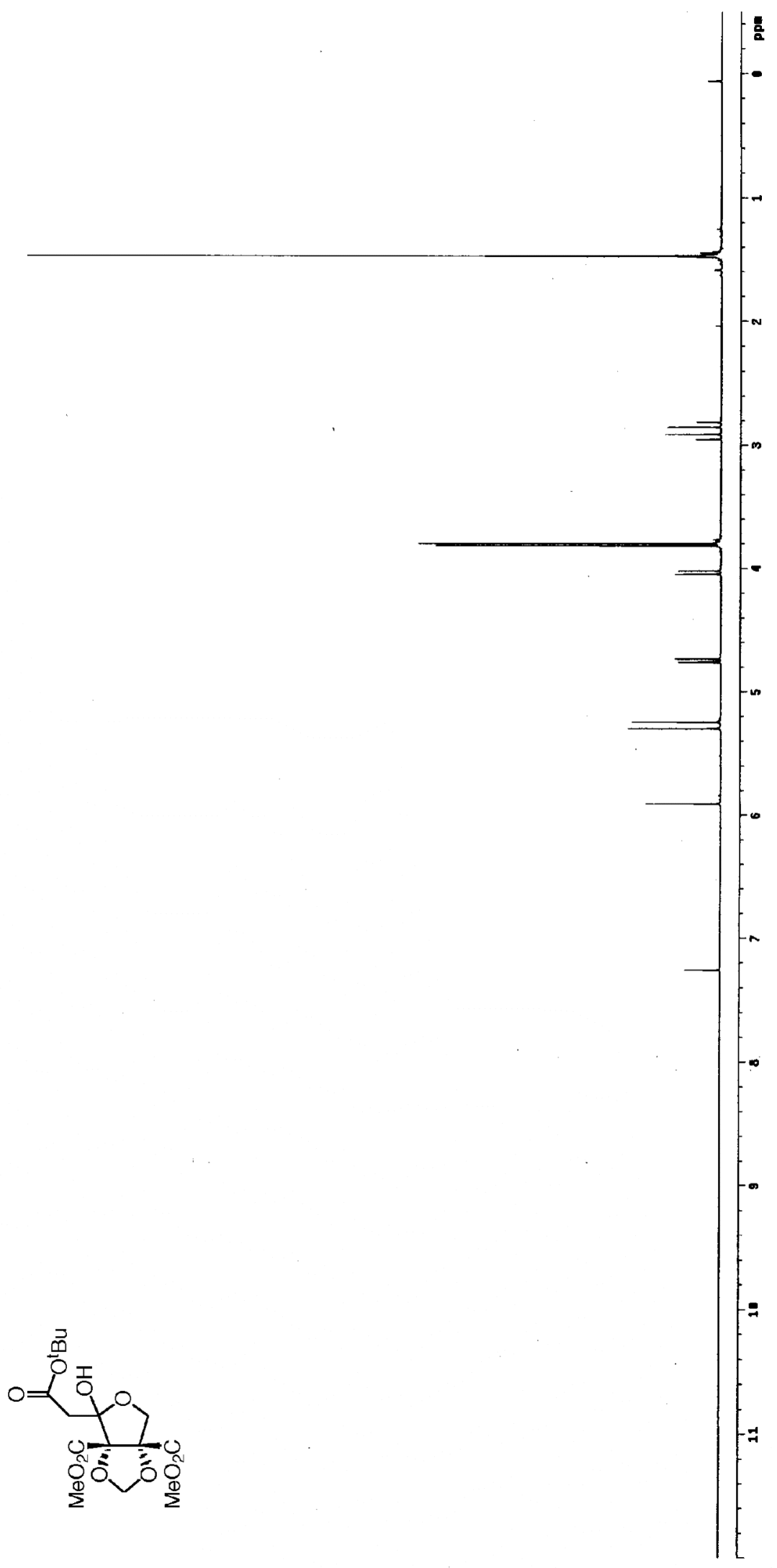


(3R-cis)-Dihydro-3,4-bis(methoxycarbonyl)-3,4-bis-(trimethyl-silanyloxy)furan-2(3H)-one.

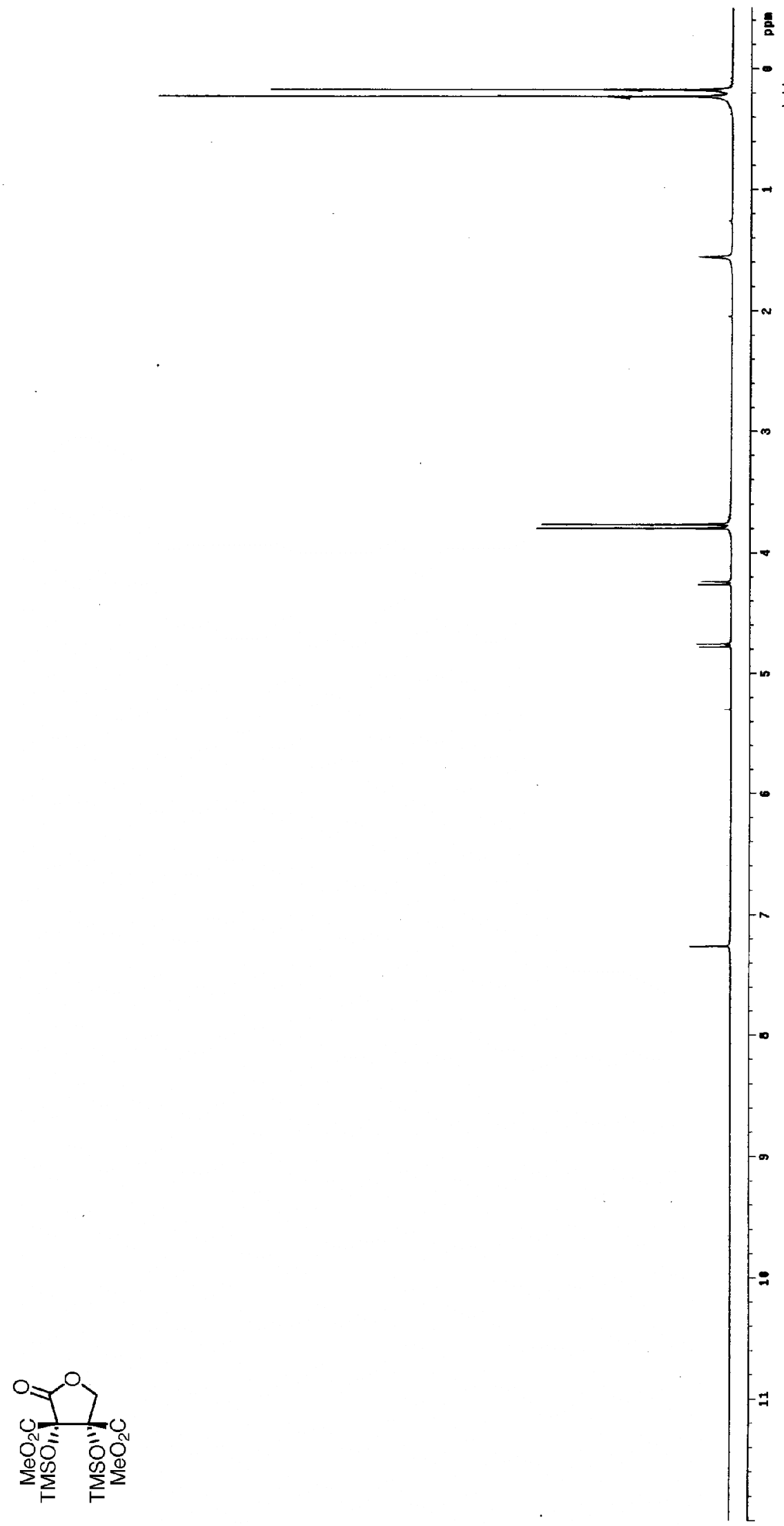


(3R-cis)-Dihydro-3,4-bis(acetoxy)-3,4-bis(methoxycarbonyl)furan-2(3H)-one.

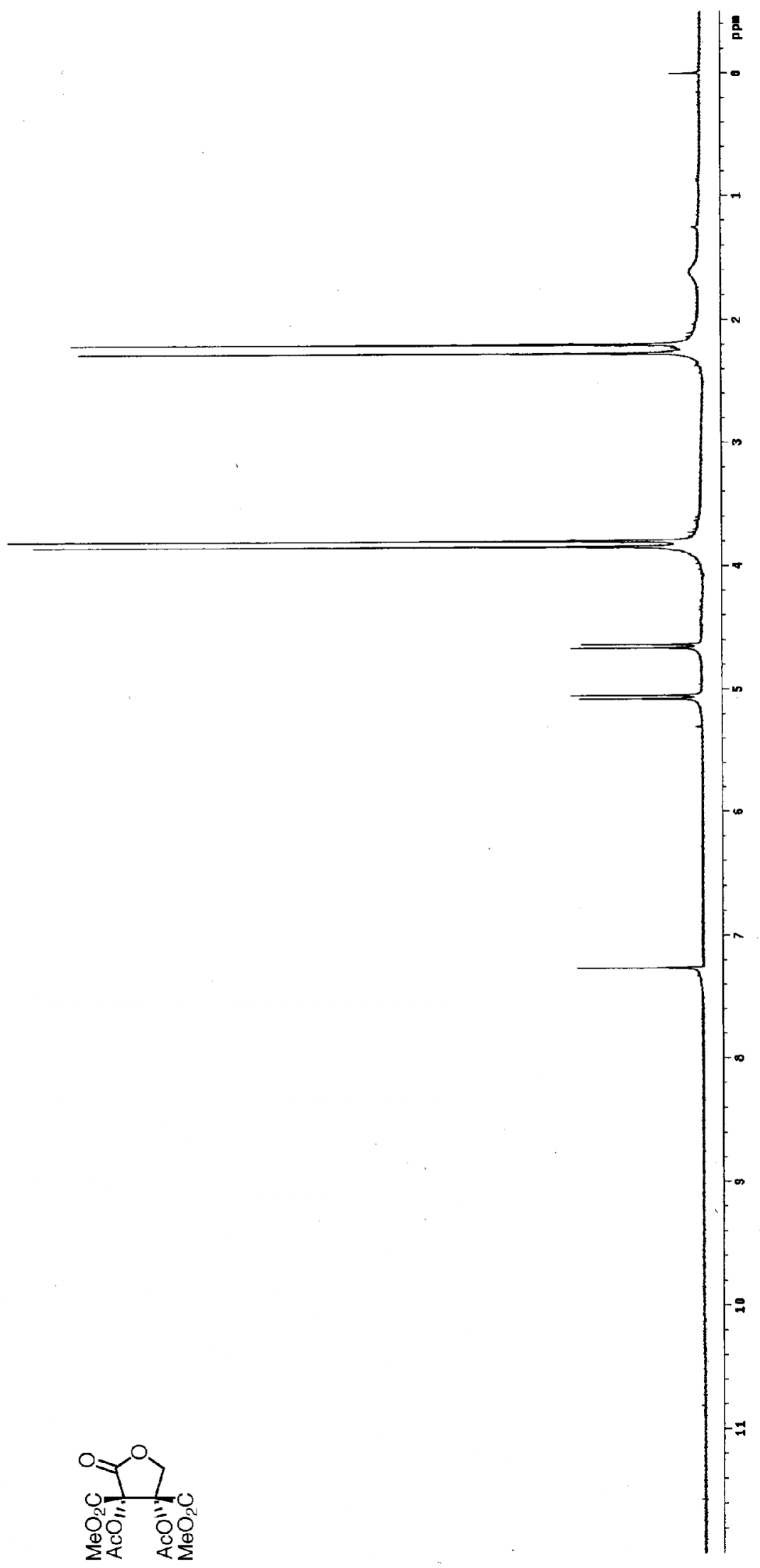


(3R-cis)-Dihydro-3,4-bis(3-bromopropionyloxy)-3,4-bis(methoxycarbonyl)furan-2(3H)-one.

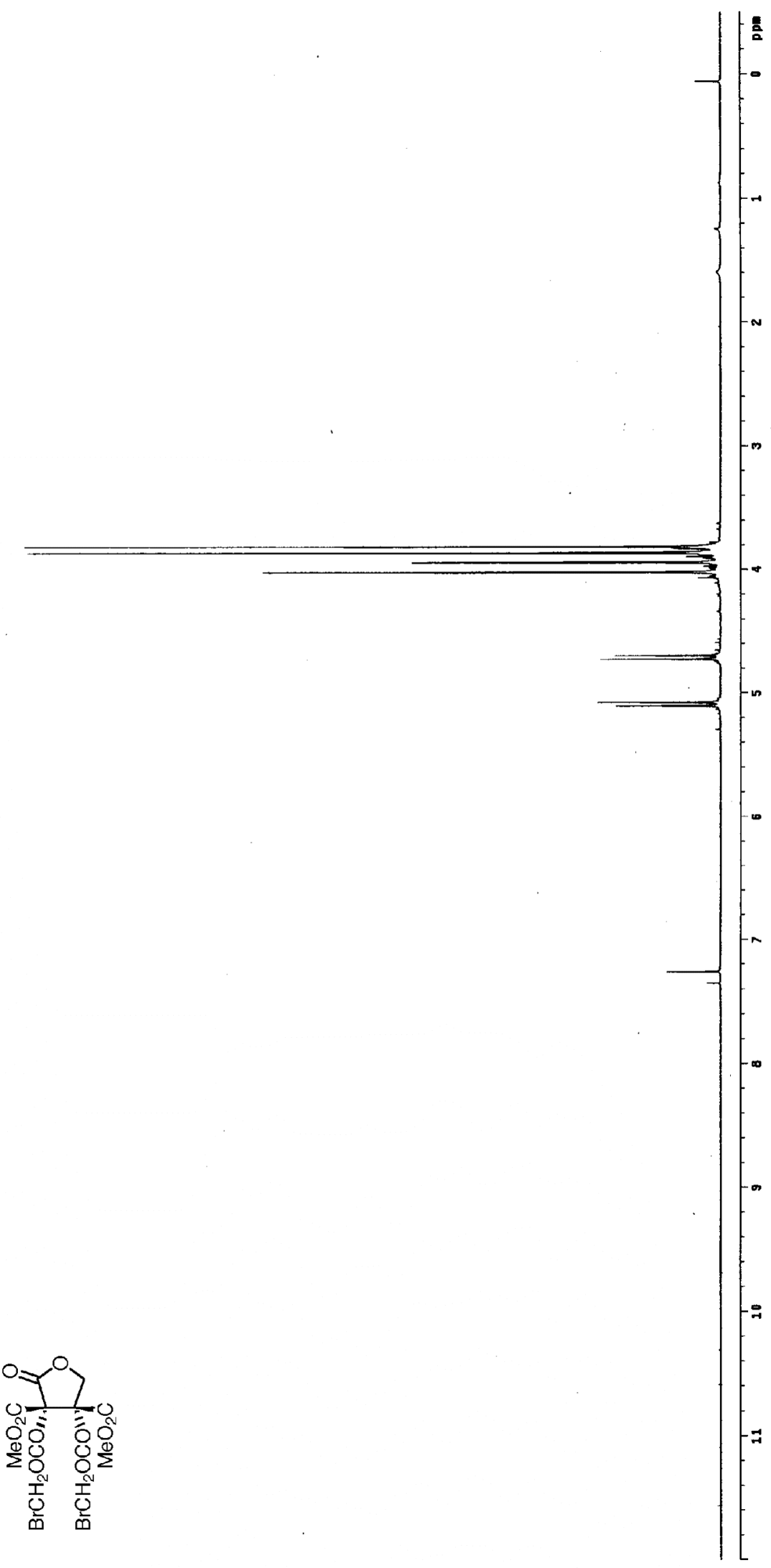

